\title{
Early science with the Large Millimetre Telescope: new mm-wave detections of circumstellar discs in IC 348 from LMT/AzTEC
}

\author{
Michael S. Petersen ${ }^{\oplus},{ }^{1 \star}$ Robert A. Gutermuth, ${ }^{1}$ Erick Nagel, ${ }^{2}$ Grant W. Wilson ${ }^{1}$ \\ and James Lane ${ }^{1,3}$ \\ ${ }^{1}$ Department of Astronomy, University of Massachusetts at Amherst, 710 N. Pleasant St, Amherst, MA 01003, USA \\ ${ }^{2}$ Department of Astronomy, Universidad de Guanajuato, Guanajuato, Gto 36240, Mexico \\ ${ }^{3}$ Physics Department, Lancaster University, Lancaster LA1 4YQ, UK
}

Accepted 2019 June 14. Received 2019 June 13; in original form 2018 November 2

\begin{abstract}
We present the most complete sample of mm measurements of protoplanetary discs in the star-forming region IC 348 to date. New observations from the Large Millimetre Telescope and the $1.1 \mathrm{~mm}$ camera AzTEC are combined with literature results in order to characterize the disc population as relating to both stellar properties within the IC 348 region and across other star-forming regions. In addition to detecting 28 of 116 observed known infrared-excess sources, we detected emission from two previously unknown candidate transition discs in the region. When combined with literature results, we find evidence for a steeper-than-expected slope, on average, in disc spectral energy distributions at millimetre wavelengths in the IC 348 region. We show that the presence or absence of high-mass discs is a sensitive indicator of regional evolution, both among star-forming regions and within IC 348. In contrast, low-mass discs exhibit almost no apparent evolution within the first $\sim 5 \mathrm{Myr}$ when compared among regions.
\end{abstract}

Key words: planets and satellites: individual: protoplanetary discs - stars: protostars - stars: rotation.

\section{INTRODUCTION}

Pre-main-sequence stars commonly possess dusty, gas-rich circumstellar discs, and it is broadly believed that these discs are the birthplaces of planets. Observations of protoplanetary circumstellar discs in the infrared reveal the presence of warm inner discs, where the accreting material for the star's continued growth resides (see Espaillat et al. 2014 for a review). However, models of infrared observations are degenerate in determining disc mass due to the optically thick nature of the hot inner disc, which complicates the determination of dust temperature, geometry, and composition (Espaillat et al. 2012). The majority of the mass is hosted in an outer disc, where large dust grains reside; the outer disc is most efficiently imaged in the (sub-)millimetre ( $\mathrm{mm}$ ) where the dust emission is generally optically thin and thus a good tracer of mass.

Over the past $10 \mathrm{yr}$, studies have sought to measure mm fluxes of protoplanetary discs to construct a timeline for circumstellar disc evolution and planet formation (see Williams \& Cieza 2011; Alexander et al. 2014, for reviews). Several regions have been studied at millimetre wavelengths, including Taurus (Andrews et al. 2013), Lupus (Ansdell et al. 2016), Chamaeleon I (Pascucci

\footnotetext{
^E-mail: Michael.Petersen@ roe.ac.uk
}

et al. 2016), $\sigma$ Ori (Ansdell et al. 2017), the Upper Scorpius OB Association (Barenfeld et al. 2016), Orion (Eisner et al. 2016, 2018), and the region studied here, IC 348 (Ruíz-Rodríguez et al. 2018). These studies have performed a linear regression to characterize a relationship between $M_{\text {dust }}$ and $M_{\star}$. Pascucci et al. (2016) compiled data from $\sim 1-3$ Myr old regions (Taurus, Lupus, and Chamaeleon I) to conclude that discs are in the fragmentationlimited regime (characterized by large pebbles undetectable in $\mathrm{mm}$ emission) owing to the steeper-than-linear relationship between $M_{\text {dust }}$ and $M_{\star}$. In this paradigm, dust evolution proceeds on a shorter time-scale around lower mass hosts. The conclusion of a steeper-than-linear $M_{\text {dust }}-M_{\star}$ relationship was bolstered by the subsequent finding of a steeper-than-linear relation in $\sigma$ Ori by Ansdell et al. (2017), albeit with large scatter $(2+$ dex $)$ in $M_{\text {dust }}$ at a given $M_{\star}$. Observations of disc sizes, while still few in number, suggest that older discs may be smaller in size than their young counterparts (Barenfeld et al. 2017), another possible explanation for the steeper-than-linear relation. However, the relationship is far from well characterized, and the simple scalings proposed deserve scrutiny. Discs can evolve based on their environment, as has been shown through disc fraction variation in the infrared (Muzerolle et al. 2010). Additionally, environmental effects are difficult to quantify, owing to the small number of regions where such studies are possible (Guarcello et al. 2016). Subdivisions of 
individual star formation regions may hold some promise in this regard.

Of the fields listed above, the young Taurus region $(t \sim 1-2 \mathrm{Myr})$ has been well studied as a result of proximity $(d \sim 140 \mathrm{pc})$, boasting a complete sample of 210 discs hosted by stars at spectral types earlier than M8.5 (Andrews \& Williams 2005, Andrews et al. 2013). However, Taurus is both young and diffuse, and generally thought to not resemble the nurseries where most stars in the Milky Way are born.

IC 348 is generally described from pre-main-sequence age estimates as a young post-embedded cluster $(t \sim 2-3 \mathrm{Myr}$, Luhman et al. 2003), though recent works have argued for an older age for IC 348 (6 Myr, Bell et al. 2013, Cottaar et al. 2014). Known to host circumstellar disc-bearing Class II young stellar objects (YSOs) by infrared analysis (Lada et al. 2006; Muench et al. 2007; Rebull et al. 2007; Jørgensen et al. 2008; Evans et al. 2009; Gutermuth et al. 2009), the evolution of the circumstellar discs in IC 348 has been probed using both disc fraction arguments as well as disc spectral energy distribution (SED) studies. The distance of IC 348, measured as $315 \mathrm{pc}$ in Muench et al. (2007), $d=273 \pm 23 \mathrm{pc}$ (Ripepi et al. 2014), $321 \pm 10$ pc from VLBA (Ortiz-León et al. 2018), and 320 pc from Gaia data (Ortiz-León et al. 2018; RuízRodríguez et al. 2018) has made the region a prime target for study and comparison with Taurus. The value of comparing to IC 348 is not just age, but also environmental difference. IC 348 is older and denser, and centred on an intermediate-mass system (HD 281159, classified as a B5 binary system in Luhman et al. 2003) whose far-ultraviolet (FUV) flux could substantially erode the outer discs of members that get too close (Adams et al. 2006). Until recently, information about disc masses in IC 348 had only been measured in 20 objects (Lee, Williams \& Cieza 2011; Espaillat et al. 2012; Cieza et al. 2015), leaving the disc mass function largely unconstrained. Andrews et al. (2013) found that with the then-current sample of disc masses in IC 348, there was no appreciable difference between Taurus and IC 348, but noted that this may be affected by the lack of completeness in the latter. With more information, Ruíz-Rodríguez et al. (2018) demonstrated a rudimentary difference between Taurus and IC 348 that they attribute to the age of the system. While RuízRodríguez et al. (2018) detected 40 discs using ALMA, more than half of the systems classified as Class II do not have detectable millimetre emission in their paper. Clearly, obtaining the most complete census of disc masses in IC 348 is then of the utmost importance for determining a possible evolutionary timeline as well as for constraining environmental impacts on disc evolution. Fully characterizing the disc population of a star-forming region such as IC 348 will add more data to constrain typical disc lifetimes, generally thought to be $\sim 3 \mathrm{Myr}$, with estimates as low as $1 \mathrm{Myr}$ and as high as $10 \mathrm{Myr}$ (Strom et al. 1989; Haisch Jr., Lada \& Lada 2001; Hillenbrand 2005; Mamajek 2009; Muzerolle et al. 2010; Pfalzner, Steinhausen \& Menten 2014).

In this paper, we present an analysis of the disc mass properties of IC 348 based on a unification of extant data in the literature with a new survey to determine the disc masses in IC 348 using the Large Millimetre Telescope (LMT) atop Volcan Sierra Negra, in Puebla, Mexico, and the $1.1 \mathrm{~mm}$ camera AzTEC (Wilson et al. 2008). In Section 2, we describe the new observations and subsequent data reduction, as well as ancillary data sources. We present results using the new data in Section 3, culminating in the most complete list of disc masses measured at $\mathrm{mm}$ wavelengths in IC 348 to date. In Section 4, we use ancillary data relating to stellar host properties to study the dependence of disc mass on stellar parameters. Section 4 focuses on the relationships between mm flux and stellar properties.
We compare our findings to the Taurus sample of Andrews et al. (2013) in Section 5 before discussing the potential implications for disc evolutionary models. We summarize in Section 6.

\section{OBSERVATIONS}

In this section, we begin by describing the target selection in IC 348. We then overview the combined AzTEC and LMT system as it pertains to observations of the IC 348 region and describe the method by which sources were extracted in Section 2.2. We describe sources of ancillary data in Section 2.3 and the matching of ancillary sources to LMT sources in Section 2.4 .

\subsection{Target selection}

Our AzTEC observations are centred on known mid-IR-excessbearing YSO targets in IC 348 from Gutermuth et al. (2009). Fig. 1 is the $\mathrm{J}$ versus $\mathrm{J}-\mathrm{H}$ colour-magnitude diagram for all such sources in IC 348, and regions are drawn with dashed lines to show mass- and extinction-limited sample areas. The original sample region named T1 requires sources with $M>0.09 \mathrm{M}_{\odot}$ (using Baraffe et al. 2015 isochrones) and $A_{V}<5$ mag. We adopted T1 for our target field selection. We successfully obtained maps of 53 of 60 target fields that covered all Gutermuth et al. (2009) identified 62 YSOs in this region. As discussed in Section 2.2 below, we expanded the masked field of view of each map in order to explore lower sensitivity areas of coverage further from the target centre point. This considerably augmented our spatial coverage, and the added areas have only modestly lower sensitivity. In total, we achieved quality detections and limits for all 62 YSOs in region T1. Furthermore, if we consider a more generous mass- and extinction-limited region of Fig. 1, such as region T2 where sources must have $M>0.05 \mathrm{M}_{\odot}$ and $A_{V}<$ $8 \mathrm{mag}$, we have detections or useful limits for 90/97 YSOs in this region, over 90 per cent coverage.

\subsection{Data acquisition}

The data were collected using the 144-element $1.1 \mathrm{~mm}$ bolometer array AzTEC (Wilson et al. 2008) on the 50-m diameter LMT during its early science phase when it was limited to a 32-m diameter collecting area. The detailed survey information is summarized in Table 1. The LMT observatory employs a dynamic queue strategy to maximize the use of AzTEC during the best observing conditions. The $\tau$ measured during our observations was 0.02 to 0.12 . We observed our targets using 'photometry mode', a Lissajous scanning pattern that is designed to produce uniform, maximal sensitivity in the central 30 arcsec area. Within this maximal sensitivity region, our detection rate of primary targets was limited. Thus, we lowered the coverage threshold in the pipeline to 10 percent of the median coverage of the map (default is 90 per cent), expanding the resultant field of view several fold and achieving reasonable sensitivity for serendipitous observations of many more disc-bearing and discless IC 348 members. The typical median noise level of the final expanded coverage maps is $\sim 1 \mathrm{mJy}$ per beam RMS, and we consistently achieve a factor of 2-4 less than that in the central high coverage zones. Noise levels are estimated directly from the time stream data using a jackknifing technique (Scott et al. 2008). AzTEC has been a guest instrument on other telescopes, and we calibrate data from AzTEC on LMT in a similar fashion to these earlier observing campaigns (e.g. Scott et al. 2008). In summary, observers obtain load curves and beam maps 2-3 times per night and pointing offset observations were taken every hour. The primary flux 
Table 1. LMT/AzTEC observations table.

\begin{tabular}{|c|c|c|c|c|c|c|}
\hline Field name & $\begin{array}{l}\text { Centre RA } \\
(\mathrm{J} 2000.0)\end{array}$ & $\begin{array}{l}\text { Centre Dec. } \\
\text { (J2000.0) }\end{array}$ & $\begin{array}{c}\text { Cov. area } \\
\text { (Sq. arcmin.) }\end{array}$ & $\begin{array}{l}\text { Median noise } \\
\left(\mathrm{mJy} \mathrm{bm}^{-1}\right)\end{array}$ & $\begin{array}{l}\text { Obs. dates } \\
\text { (YMD) }\end{array}$ & Tau $(225 \mathrm{GHz})$ \\
\hline IC348-Field01 & 034433.79 & +315830.2 & 13.28 & 1.45 & 2014-12-08 & 0.07 \\
\hline IC348-Field02 & 034415.84 & +315936.8 & 13.72 & 1.46 & 2014-12-09 & 0.06 \\
\hline IC348-Field03 & 034428.51 & +315953.9 & 13.74 & 5.54 & 2014-12-06 & 0.08 \\
\hline IC348-Field04 & 034535.63 & +315954.5 & 13.87 & 1.46 & $\begin{array}{l}2014-12-09 \\
2014-12-17\end{array}$ & $\begin{array}{l}0.06 \\
0.10\end{array}$ \\
\hline IC348-Field05 & 034429.82 & +320054.6 & 13.31 & 1.71 & 2014-12-09 & 0.06 \\
\hline IC348-Field06 & 034328.20 & +320159.2 & 13.52 & 1.37 & 2014-12-09 & 0.06 \\
\hline IC348-Field07 & 034437.98 & +320329.6 & 13.13 & 1.43 & 2014-12-09 & 0.07 \\
\hline IC348-Field08 & 034444.72 & +320402.5 & 12.51 & 1.52 & 2014-12-09 & 0.07 \\
\hline IC348-Field09 & 034410.13 & +320404.5 & 11.62 & 1.24 & 2014-12-09 & 0.07 \\
\hline IC348-Field10 & 034426.03 & +320430.3 & 11.42 & 1.56 & 2014-12-11 & 0.06 \\
\hline IC348-Field11 & 034520.46 & +320634.5 & 11.86 & 2.05 & 2014-12-17 & 0.10 \\
\hline IC348-Field12 & 034436.95 & +320645.3 & 11.09 & 1.68 & 2014-12-17 & 0.10 \\
\hline IC348-Field13 & 034438.46 & +320735.8 & 10.86 & 2.23 & 2014-12-17 & 0.10 \\
\hline IC348-Field15 & 034355.27 & +320753.3 & 11.59 & 0.94 & 2014-12-19 & 0.07 \\
\hline IC348-Field17 & 034442.76 & +320833.8 & 11.06 & 1.13 & 2014-12-19 & 0.07 \\
\hline IC348-Field19 & 034420.18 & +320856.6 & 12.33 & 1.52 & 2015-01-13 & 0.03 \\
\hline IC348-Field21 & 034456.15 & +3209 15.2 & 12.24 & 1.19 & 2015-01-13 & 0.03 \\
\hline IC348-Field22 & 034435.96 & +320924.4 & 11.88 & 1.06 & 2015-01-13 & 0.03 \\
\hline IC348-Field23 & 034525.15 & +320930.3 & 11.51 & 1.10 & 2015-01-13 & 0.03 \\
\hline IC348-Field27 & 034418.19 & +320959.3 & 11.05 & 1.49 & 2015-01-13 & 0.03 \\
\hline IC348-Field29 & 034425.30 & +321012.8 & 10.88 & 1.53 & 2015-01-13 & 0.03 \\
\hline IC348-Field30 & 034443.76 & +32 1030.4 & 10.90 & 0.89 & $\begin{array}{l}2015-01-14 \\
2015-01-15\end{array}$ & $\begin{array}{l}0.05 \\
0.06\end{array}$ \\
\hline IC348-Field34 & 034431.35 & +321047.0 & 11.27 & 1.41 & $\begin{array}{l}2015-01-15 \\
2015-01-16\end{array}$ & $\begin{array}{l}0.06 \\
0.07\end{array}$ \\
\hline IC348-Field35 & 034358.91 & +321127.1 & 11.77 & 1.13 & 2015-01-16 & 0.06 \\
\hline IC348-Field38 & 034517.82 & +321205.9 & 11.50 & 1.02 & 2015-01-16 & 0.06 \\
\hline IC348-Field39 & 034437.39 & +32 1224.2 & 12.44 & 2.46 & 2015-02-02 & 0.10 \\
\hline IC348-Field40 & 034323.55 & +32 1225.9 & 12.27 & 1.38 & 2015-02-02 & 0.10 \\
\hline IC348-Field41 & 034421.28 & +32 1237.2 & 11.39 & 1.26 & 2015-02-02 & 0.10 \\
\hline IC348-Field42 & 034427.25 & +321421.0 & 11.31 & 1.21 & 2015-02-05 & 0.07 \\
\hline IC348-Field43 & 034359.08 & +321421.4 & 11.09 & 1.27 & 2015-02-05 & 0.07 \\
\hline IC348-Field44 & 034434.99 & +32 1531.2 & 12.39 & 1.73 & 2015-02-06 & 0.05 \\
\hline IC348-Field45 & 034348.82 & +3215 51.6 & 11.02 & 1.11 & 2015-02-06 & 0.03 \\
\hline IC348-Field46 & 034443.02 & +3215 59.6 & 11.61 & 1.30 & 2015-02-07 & 0.06 \\
\hline IC348-Field47 & 034434.12 & +321635.8 & 11.15 & 1.07 & 2015-02-07 & 0.06 \\
\hline IC348-Field48 & 034358.57 & +32 1727.6 & 11.44 & 1.37 & 2015-02-07 & 0.06 \\
\hline IC348-Field49 & 034439.79 & +32 1804.1 & 11.87 & 1.33 & 2015-02-08 & 0.09 \\
\hline IC348-Field50 & 034415.23 & +32 1942.3 & 11.33 & 0.87 & $\begin{array}{l}2015-02-08 \\
2015-02-10\end{array}$ & $\begin{array}{l}0.11 \\
0.07\end{array}$ \\
\hline IC348-Field51 & 034439.19 & +32 2008.9 & 10.95 & 0.84 & $\begin{array}{l}2015-02-08 \\
2015-02-10\end{array}$ & $\begin{array}{l}0.12 \\
0.05\end{array}$ \\
\hline IC348-Field52 & 034427.21 & +322028.8 & 10.87 & 0.89 & $\begin{array}{l}2015-02-08 \\
2015-02-10\end{array}$ & $\begin{array}{l}0.11 \\
0.05\end{array}$ \\
\hline IC348-Field53 & 034513.50 & +322434.7 & 10.85 & 1.40 & 2015-02-10 & 0.05 \\
\hline IC348-Field54 & 034422.58 & +320153.7 & 11.37 & 1.17 & $\begin{array}{l}2015-03-03 \\
2015-03-04\end{array}$ & $\begin{array}{l}0.02 \\
0.02\end{array}$ \\
\hline IC348-Field56 & 034406.79 & +320754.1 & 10.85 & 1.23 & $\begin{array}{l}2015-03-04 \\
2015-03-06\end{array}$ & $\begin{array}{l}0.02 \\
0.04\end{array}$ \\
\hline IC348-Field57 & 034344.61 & +320817.8 & 11.31 & 2.43 & 2015-03-06 & 0.05 \\
\hline
\end{tabular}

calibrator and beam-mapping target was CRL618, and the pointing offset calibrator target was $3 \mathrm{c} 111$ for all fields. Pointing offsets drifted by $1.5-2$ arcsec per hour. We interpolate pointing offsets between successive calibrator measurements, thus residual pointing offsets should be $<1$ arcsec.

All the data were processed following the standard AzTEC data treatment, including 'despiking' of the signal time streams, lowpass filtering, and principal component analysis (PCA) atmospheric filtering (Scott et al. 2008) as implemented in the standard AzTEC
$\mathrm{C}++$ data reduction pipeline. The final maps are produced by applying adaptive Wiener filtering to the data. The goal is to produce and apply an optimized filter for detecting unresolved sources without sacrificing the excellent angular resolution of AzTEC and LMT (8.5 arcsec at 32-m diameter). The depth of our maps revealed a fair amount of residual low column density cloud structure, and the high default setting for the PCA filtering and the Wiener filtering created numerous false point sources within it. To attempt to more clearly distinguish between bona fide point sources and strongly 


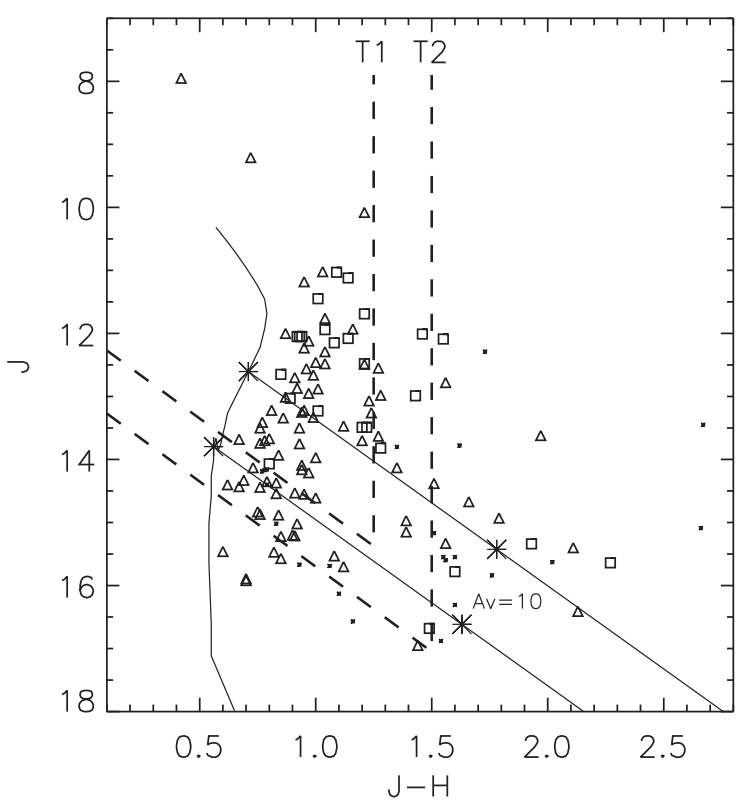

Figure 1. J versus $\mathrm{J}-\mathrm{H}$ diagram for the YSO members of IC 348 that possess circumstellar discs as identified in Gutermuth et al. (2009). Objects with successful mm detections with LMT/AzTEC are plotted as squares, and those with quality upper limits are plotted as triangles. The small plot points mark those objects that were not covered by the LMT in our survey. For reference, we overlay the data with the $3 \mathrm{Myr}$ pre-main-sequence stellar model isochrone of Baraffe et al. (2015) that spans masses of 0.01-1.4 $\mathrm{M}_{\odot}$ (curved solid line) and extinction vectors from Rieke \& Lebofsky (1985) (straight solid lines with large asterisks at $A_{V}=0$ and $10 \mathrm{mag}$ ) extending from masses of 0.3 and $0.08 \mathrm{M}_{\odot}$. The dashed lines mark the mass- and extinction-limited target sample areas $\mathrm{T} 1$ and $\mathrm{T} 2$, for reference.

filtered resolved emission, we performed a secondary filtering process on all the data, replacing the Wiener filter with a simple Gaussian filter of the same full width at half-maximum (FWHM) as the telescope beam ( 8.5 arcsec) and reducing the strength of the PCA filter to 3 from 9 eigenvectors. This treatment, named 'soft filtering' below to distinguish it from the 'hard filtering' of the standard process, is not optimized for point sources, and thus it does not achieve as low noise levels and it yields a marginal loss of angular resolution in the final images (12 arcsec FWHM). However, it is far less destructive to resolved emission, facilitating a fair evaluation of the nature of sources detected in both treatments.

We identify and extract point sources in the same manner as pre-stellar dense gas cores were extracted in another AzTEC/LMT survey (Sokol et al. 2019). In summary, each flux map is masked to include those pixels that have sufficient $\mathrm{S} / \mathrm{N}$ and coverage (2.5 and 10 per cent of median coverage, respectively), scaled by its median noise value, inverted, and fed to the native IDL function watershed to identify and merge regions of contiguous emission with some central peak. Saddle points in the flux distribution are also identified and used to split close sources. Reported positions are flux-weighted means of the pixels within the half-peak-power emission area, and point source fluxes and $\mathrm{S} / \mathrm{N}$ are obtained from the locations of the observed peak flux values within each source footprint. We reject all source candidates whose footprints fall within 8 arcsec of the coverage edge to eliminate a large source of atmospheric filtration artefacts. The process to extract resolved structures from the soft filtering process is identical.
Once we have source catalogues derived from the two filtering methods' images, the positions of all prospective point sources detected with strong filtering are checked for a resolved source in the soft filtering list. Where no match is found, an upper limit is extracted to ensure consistency with that source being lost in the elevated noise of the soft filtering. Where matches exist, source concentration (peak to total flux ratio, including a low S/N correction; Sokol et al., in preparation) derived from the soft filtering data and ratios of the peak fluxes from both methods are computed. We adopt an empirical limit that any object with a soft filtering counterpart must also have a peak to total flux ratio greater than 0.4 , or else we consider that detection to be cloud-dominated and thus a poor representation of the disc flux. Where objects are clouddominated, the hard filtering flux is instead reported as an upper limit set by our inability to separate disc from cloud emission with confidence rather than by noise constraints. For all YSOs that have no detections under soft or hard filtering, we report three times the noise value of the nearest pixel position as our noise-bound upper limit.

Using the YSO list of Gutermuth et al. (2009), we found that 116 total source positions had been covered, of the complete sample of 160 in Gutermuth et al. (2009). We extracted sources where possible, and measured upper limits at the expected source position for those not detected. We detected 28 sources, with 88 additional upper limits. Seven sources were detected in multiple fields that had serendipitous overlapping coverage. 15 additional sources were detected once and had an additional upper limit in coverage from another map. The data are given in Table 2. We list the statistics for the LMT-detected sources from Gutermuth et al. (2009) in the upper frame of Table 3.

\subsection{Ancillary data}

While mm points have been used in previous studies as a direct mapping to disc mass, ancillary data available for IC 348 allow for a more robust characterization of sources, as well as potentially providing valuable information about the evolutionary behaviour of circumstellar discs. We use infrared YSO determinations and infrared photometry from Gutermuth et al. (2009) and corresponding stellar properties from Cottaar et al. (2014), briefly describing the data below.

\subsubsection{Infrared YSO identification}

IC 348 has been well studied in the infrared. Four-band Spitzer Infrared Array Camera (IRAC, at 3.6, 4.5, 5.8, 8.0 $\mu \mathrm{m}$ ) and Multiband Imaging Photometer System (MIPS, at $24 \mu \mathrm{m}$ ) were obtained and presented by Lada et al. (2006). The data were subsequently examined by Jørgensen et al. (2006), Rebull et al. (2007), Muench et al. (2007), Evans et al. (2009), and Gutermuth et al. (2009). Gutermuth et al. (2009) reduced IC 348 as part of a large survey of nearby star-forming clusters and groups, finding 160 YSOs in IC 348 (compared to 189 in Jørgensen et al. 2008 and 156 in the spectroscopicically informed study of Muench et al. 2007, with minor classification technique discrepancies attributed to differing fields of view), 16 of which are Class I sources (compared to 15 in Jørgensen et al. 2008 and 20 in Muench et al. 2007). Identified YSOs were matched to 2MASS (Skrutskie et al. 2006) sources, providing $J H K_{S}$ photometry. We adopt the classifications of Gutermuth et al. (2009) here, noting that minor differences exist between that source list and others for IC 348, which we address below where relevant. 
Table 2. IR excess matched sources. Entries with -1 indicate that we have no detections. Sources 49, 59, and 70 potentially contain flux from nearby cloud material. We mark these fluxes with a '*' and treat them as upper limits in the statistical analysis below. Class I* sources, following the nomenclature of Gutermuth et al. (2009), are 'deeply embedded' Class I sources. IR excess matched sources. Class II* sources, following the nomenclature of Gutermuth et al. (2009), are transition disc (TD) candidates.

\begin{tabular}{|c|c|c|c|c|c|c|c|c|}
\hline G09 & $\begin{array}{l}\text { RA (G09) } \\
\quad(\mathrm{deg})\end{array}$ & $\begin{array}{l}\text { Dec. (G09) } \\
\quad(\mathrm{deg})\end{array}$ & $\begin{array}{c}\delta \mathrm{RA} \\
(\operatorname{arcsec})\end{array}$ & $\begin{array}{l}\delta \text { Dec. } \\
(\operatorname{arcsec})\end{array}$ & $\begin{array}{l}\text { Flux } \\
(\mathrm{mJy})\end{array}$ & $\mathrm{SN}$ & $\begin{array}{l}\mathrm{T}_{\star} \\
(\mathrm{K})\end{array}$ & Class \\
\hline 4 & 56.10358 & 32.23000 & 0.0 & 0.0 & 2.76 & -1.00 & $-1.00 \pm-1.00$ & $\mathrm{I}^{*}$ \\
\hline 12 & 56.03833 & 32.04381 & 2.6 & 3.5 & 9.22 & 8.06 & $4106.23 \pm 58.13$ & I \\
\hline 14 & 56.12625 & 32.19314 & 0.0 & 0.0 & 3.18 & -1.00 & $-1.00 \pm-1.00$ & I \\
\hline 16 & 56.30758 & 32.20278 & 5.0 & -2.5 & 7.51 & 10.31 & $-1.00 \pm-1.00$ & I \\
\hline 18 & 55.84813 & 32.20719 & 0.0 & 0.0 & 2.16 & -1.00 & $3087.79 \pm 12.62$ & II \\
\hline 19 & 55.86750 & 32.03311 & 0.0 & 0.0 & 1.95 & -1.00 & $3789.68 \pm 4.84$ & II \\
\hline 23 & 55.94850 & 32.15072 & 0.0 & 0.0 & 5.79 & -1.00 & $2654.35 \pm 45.64$ & II \\
\hline 24 & 55.95342 & 32.26433 & 0.0 & 0.0 & 1.80 & -1.00 & $3464.19 \pm 5.74$ & II \\
\hline 26 & 55.98029 & 32.13147 & 0.0 & 0.0 & 1.50 & -1.00 & $2711.48 \pm 31.18$ & II \\
\hline 30 & 55.98492 & 32.16647 & 0.0 & 0.0 & 4.59 & -1.00 & $2139.14 \pm 0.55$ & II \\
\hline 32 & 55.99404 & 32.29100 & -0.1 & -1.7 & 3.41 & 4.44 & $3369.24 \pm 4.43$ & II \\
\hline 33 & 55.99546 & 32.19086 & 1.4 & -1.6 & 2.01 & 3.44 & $3701.93 \pm 4.88$ & II \\
\hline 34 & 55.99617 & 32.23928 & 1.8 & 0.2 & 3.66 & 5.30 & $3156.47 \pm 28.06$ & II \\
\hline 41 & 56.02500 & 32.25894 & 0.0 & 0.0 & 6.93 & -1.00 & $2682.95 \pm 29.85$ & II \\
\hline 43 & 56.04221 & 32.06792 & 0.0 & 0.0 & 1.98 & -1.00 & $2868.23 \pm 17.81$ & II \\
\hline 45 & 56.04792 & 32.30264 & 0.0 & 0.0 & 4.26 & -1.00 & $-1.00 \pm-1.00$ & II \\
\hline 46 & 56.04842 & 32.05364 & 0.0 & 0.0 & 2.88 & -1.00 & $6140.89 \pm 26.31$ & II \\
\hline 47 & 56.05408 & 32.22097 & 0.0 & 0.0 & 8.37 & -1.00 & $2937.47 \pm 18.88$ & II \\
\hline 48 & 56.06346 & 32.32842 & 0.0 & 0.0 & 1.41 & -1.00 & $-1.00 \pm-1.00$ & II \\
\hline 49 & 56.06600 & 31.99356 & -1.2 & 0.0 & $4.24^{\star}$ & 6.04 & $2842.07 \pm 21.67$ & II \\
\hline 50 & 56.07537 & 32.18153 & 0.0 & 0.0 & 3.57 & -1.00 & $4067.21 \pm 50.96$ & II \\
\hline 51 & 56.07571 & 32.08253 & 3.1 & -0.9 & 31.20 & 10.28 & $5318.05 \pm 28.09$ & II \\
\hline 52 & 56.07579 & 32.16647 & 0.0 & 0.0 & 2.40 & -1.00 & $3204.55 \pm 22.33$ & II \\
\hline 53 & 56.07746 & 32.21478 & 1.4 & 0.4 & 2.87 & 3.24 & $2652.84 \pm 79.92$ & II \\
\hline 54 & 56.08017 & 32.12628 & 0.0 & 0.0 & 4.17 & -1.00 & $3516.26 \pm 16.30$ & II \\
\hline 55 & 56.08408 & 32.14906 & 0.0 & 0.0 & 2.25 & -1.00 & $3351.63 \pm 10.00$ & II \\
\hline 56 & 56.08842 & 32.02067 & 0.0 & 0.0 & 2.58 & -1.00 & $-1.00 \pm-1.00$ & II \\
\hline 57 & 56.08867 & 32.21033 & 0.0 & 0.0 & 2.04 & -1.00 & $3046.85 \pm 27.42$ & II \\
\hline 58 & 56.08871 & 32.19900 & 0.0 & 0.0 & 2.58 & -1.00 & $3716.55 \pm 10.36$ & II \\
\hline 59 & 56.08896 & 31.99239 & -3.8 & -2.2 & $13.46^{\star}$ & 11.26 & $1529.32 \pm 1.48$ & II \\
\hline 60 & 56.09008 & 32.17714 & 0.0 & 0.0 & 3.45 & -1.00 & $3901.05 \pm 11.28$ & II \\
\hline 62 & 56.09287 & 32.09522 & 0.0 & 0.0 & 5.10 & -1.00 & $4079.71 \pm 12.32$ & II \\
\hline 63 & 56.09300 & 32.20019 & 0.0 & 0.0 & 2.55 & -1.00 & $3701.25 \pm 24.66$ & II \\
\hline 64 & 56.09458 & 32.02842 & 0.0 & 0.0 & 1.86 & -1.00 & $-1.00 \pm-1.00$ & II \\
\hline 65 & 56.09558 & 32.24461 & 0.0 & 0.0 & 2.88 & -1.00 & $-1.00 \pm-1.00$ & II \\
\hline 66 & 56.09821 & 32.15939 & 0.0 & 0.0 & 3.39 & -1.00 & $2906.62 \pm 26.26$ & II \\
\hline 67 & 56.09850 & 32.03131 & 0.0 & 0.0 & 1.89 & -1.00 & $-1.00 \pm-1.00$ & II \\
\hline 68 & 56.10192 & 32.02883 & 0.0 & 0.0 & 1.98 & -1.00 & $-1.00 \pm-1.00$ & II \\
\hline 69 & 56.10542 & 32.17022 & 0.0 & 0.0 & 2.55 & -1.00 & $3051.45 \pm 16.40$ & II \\
\hline 70 & 56.10637 & 32.19194 & -1.5 & 0.6 & $6.44^{\star}$ & 3.93 & $3865.53 \pm 25.97$ & II \\
\hline 71 & 56.10708 & 32.26369 & 0.0 & 0.0 & 4.44 & -1.00 & $-1.00 \pm-1.00$ & II \\
\hline 72 & 56.10846 & 32.07508 & 0.0 & 0.0 & 2.46 & -1.00 & $4680.76 \pm 13.35$ & II \\
\hline 73 & 56.11121 & 32.13897 & 4.0 & 1.0 & 4.74 & 3.40 & $3782.54 \pm 11.35$ & II \\
\hline 74 & 56.11112 & 32.04342 & 0.0 & 0.0 & 2.94 & -1.00 & $-1.00 \pm-1.00$ & II \\
\hline 75 & 56.11337 & 32.34133 & 0.0 & 0.0 & 1.47 & -1.00 & $2955.00 \pm 10.36$ & II \\
\hline 76 & 56.11354 & 32.17703 & 0.0 & 0.0 & 2.91 & -1.00 & $2986.15 \pm 33.23$ & II \\
\hline 77 & 56.11354 & 32.23917 & 0.0 & 0.0 & 1.95 & -1.00 & $3375.08 \pm 5.81$ & II \\
\hline 78 & 56.11879 & 31.99831 & 0.0 & 0.0 & 3.84 & -1.00 & $3537.16 \pm 7.41$ & II \\
\hline 79 & 56.12062 & 32.02717 & -2.9 & 2.2 & 3.77 & 3.96 & $3594.34 \pm 38.74$ & II \\
\hline 80 & 56.12179 & 32.02106 & 0.0 & 0.0 & 2.67 & -1.00 & $-1.00 \pm-1.00$ & II \\
\hline 81 & 56.12387 & 32.17775 & 0.0 & 0.0 & 2.70 & -1.00 & $4045.34 \pm 11.51$ & II \\
\hline 82 & 56.12425 & 32.01517 & 0.0 & 0.0 & 2.49 & -1.00 & $2735.18 \pm 20.87$ & II \\
\hline 83 & 56.12488 & 32.16097 & 0.0 & 0.0 & 3.30 & -1.00 & $-1.00 \pm-1.00$ & II \\
\hline 84 & 56.12554 & 32.02172 & 0.0 & 0.0 & 2.70 & -1.00 & $3690.22 \pm 40.69$ & II \\
\hline 85 & 56.12896 & 32.04561 & 0.0 & 0.0 & 4.71 & -1.00 & $3044.82 \pm 162.39$ & II \\
\hline 86 & 56.12975 & 32.31347 & 0.0 & 0.0 & 5.34 & -1.00 & $-1.00 \pm-1.00$ & II \\
\hline 87 & 56.12996 & 32.09972 & -3.5 & -0.0 & 6.80 & 3.08 & $3344.95 \pm 143.18$ & II \\
\hline 88 & 56.13062 & 32.17972 & 0.0 & 0.0 & 2.31 & -1.00 & $3301.95 \pm 15.83$ & II \\
\hline 89 & 56.13071 & 32.00389 & 0.0 & 0.0 & 3.06 & -1.00 & $3794.67 \pm 19.18$ & II \\
\hline 90 & 56.13488 & 32.05761 & 0.0 & 0.0 & 3.51 & -1.00 & $-1.00 \pm-1.00$ & II \\
\hline
\end{tabular}


Table 2 - continued

\begin{tabular}{|c|c|c|c|c|c|c|c|c|}
\hline G09 & $\begin{array}{c}\text { RA (G09) } \\
\text { (deg) }\end{array}$ & $\begin{array}{c}\text { Dec. (G09) } \\
\quad(\operatorname{deg})\end{array}$ & $\begin{array}{c}\delta \mathrm{RA} \\
(\operatorname{arcsec})\end{array}$ & $\begin{array}{l}\delta \text { Dec. } \\
(\operatorname{arcsec})\end{array}$ & $\begin{array}{l}\text { Flux } \\
\text { (mJy) }\end{array}$ & $\mathrm{SN}$ & $\begin{array}{l}\mathrm{T}_{\star} \\
(\mathrm{K})\end{array}$ & Class \\
\hline 91 & 56.13837 & 32.21597 & 0.0 & 0.0 & 5.94 & -1.00 & $3105.58 \pm 71.12$ & II \\
\hline 92 & 56.14079 & 31.97506 & 2.5 & -0.4 & 12.16 & 6.82 & $3347.68 \pm 5.58$ & II \\
\hline 93 & 56.14179 & 32.11583 & 0.0 & 0.0 & 3.63 & -1.00 & $-1.00 \pm-1.00$ & II \\
\hline 94 & 56.14217 & 32.27661 & 0.0 & 0.0 & 1.80 & -1.00 & $3082.04 \pm 32.69$ & II \\
\hline 95 & 56.14283 & 32.21131 & 0.0 & 0.0 & 5.01 & -1.00 & $2205.29 \pm 0.00$ & II \\
\hline 96 & 56.14454 & 32.26672 & 2.3 & -0.8 & 10.29 & 12.55 & $-1.00 \pm-1.00$ & II \\
\hline 97 & 56.14579 & 32.25867 & 0.0 & 0.0 & 2.52 & -1.00 & $3393.35 \pm 15.37$ & II \\
\hline 98 & 56.14587 & 32.14928 & 0.0 & 0.0 & 1.86 & -1.00 & $-1.00 \pm-1.00$ & II \\
\hline 99 & 56.14733 & 32.16794 & 0.0 & 0.0 & 2.40 & -1.00 & $6754.92 \pm 2.77$ & II \\
\hline 100 & 56.14742 & 32.12672 & 3.2 & -1.0 & 8.02 & 5.45 & $3891.78 \pm 8.66$ & II \\
\hline 101 & 56.14775 & 32.14897 & 0.0 & 0.0 & 1.77 & -1.00 & $2887.69 \pm 22.63$ & II \\
\hline 102 & 56.14867 & 32.05100 & 0.0 & 0.0 & 2.55 & -1.00 & $-1.00 \pm-1.00$ & II \\
\hline 103 & 56.14975 & 32.18819 & 0.0 & 0.0 & 3.39 & -1.00 & $-1.00 \pm-1.00$ & II \\
\hline 104 & 56.14983 & 32.15678 & 0.0 & 0.0 & 1.62 & -1.00 & $-1.00 \pm-1.00$ & II \\
\hline 105 & 56.15396 & 32.11258 & 0.0 & 0.0 & 2.76 & -1.00 & $5274.51 \pm 7.51$ & II \\
\hline 106 & 56.15588 & 32.15025 & 0.0 & 0.0 & 1.77 & -1.00 & $-1.00 \pm-1.00$ & II \\
\hline 107 & 56.15579 & 32.20672 & 0.0 & 0.0 & 3.75 & -1.00 & $3702.43 \pm 14.33$ & II \\
\hline 108 & 56.15825 & 32.05822 & -0.3 & -1.3 & 2.60 & 3.70 & $4144.64 \pm 6.66$ & II \\
\hline 109 & 56.15833 & 32.19361 & 0.0 & 0.0 & 4.62 & -1.00 & $3211.30 \pm 23.40$ & II \\
\hline 110 & 56.15896 & 32.17267 & 0.0 & 0.0 & 2.52 & -1.00 & $-1.00 \pm-1.00$ & II \\
\hline 111 & 56.16025 & 32.12661 & 0.0 & 0.0 & 3.54 & -1.00 & $4327.22 \pm 23.13$ & II \\
\hline 112 & 56.16063 & 32.13350 & -4.8 & 2.3 & 7.35 & 7.07 & $3887.56 \pm 12.84$ & II \\
\hline 113 & 56.16237 & 32.05544 & 0.0 & 0.0 & 2.25 & -1.00 & $-1.00 \pm-1.00$ & II \\
\hline 114 & 56.16329 & 32.33581 & 4.5 & -1.9 & 1.53 & 3.33 & $3127.66 \pm 5.34$ & II \\
\hline 115 & 56.16333 & 32.16247 & 0.0 & 0.0 & 2.13 & -1.00 & $3721.75 \pm 20.32$ & II \\
\hline 116 & 56.16579 & 32.30114 & 0.0 & 0.0 & 2.10 & -1.00 & $3318.68 \pm 5.06$ & II \\
\hline 117 & 56.16762 & 32.15919 & 0.0 & 0.0 & 2.28 & -1.00 & $3112.63 \pm 38.09$ & II \\
\hline 118 & 56.17171 & 32.10750 & 0.0 & 0.0 & 3.75 & -1.00 & $2879.87 \pm 64.61$ & II \\
\hline 119 & 56.17325 & 32.17761 & 0.0 & 0.0 & 1.92 & -1.00 & $-1.00 \pm-1.00$ & II \\
\hline 120 & 56.17554 & 32.15050 & -0.9 & -4.1 & 3.60 & 3.47 & $-1.00 \pm-1.00$ & II \\
\hline 121 & 56.17737 & 32.16736 & 0.0 & 0.0 & 1.77 & -1.00 & $3119.63 \pm 13.47$ & II \\
\hline 122 & 56.17817 & 32.14272 & 3.0 & -1.4 & 7.32 & 6.82 & $3186.70 \pm 20.17$ & II \\
\hline 123 & 56.17925 & 32.26656 & 0.0 & 0.0 & 2.10 & -1.00 & $3010.11 \pm 25.53$ & II \\
\hline 124 & 56.18233 & 32.17511 & 0.0 & 0.0 & 1.50 & -1.00 & $4028.92 \pm 11.89$ & II \\
\hline 125 & 56.18575 & 32.13681 & 0.0 & 0.0 & 2.07 & -1.00 & $3651.96 \pm 12.26$ & II \\
\hline 126 & 56.18633 & 32.06736 & 3.4 & -2.4 & 2.43 & 3.15 & $3723.36 \pm 9.46$ & II \\
\hline 128 & 56.18846 & 32.18211 & 0.0 & 0.0 & 1.71 & -1.00 & $-1.00 \pm-1.00$ & II \\
\hline 129 & 56.18979 & 32.30553 & 0.0 & 0.0 & 3.30 & -1.00 & $3972.21 \pm 91.82$ & II \\
\hline 130 & 56.19138 & 32.06575 & 0.0 & 0.0 & 2.43 & -1.00 & $-1.00 \pm-1.00$ & II \\
\hline 133 & 56.23396 & 32.15422 & 0.0 & 0.0 & 1.80 & -1.00 & $4409.40 \pm 8.32$ & II \\
\hline 143 & 56.30625 & 32.40964 & 0.0 & 0.0 & 2.28 & -1.00 & $3103.29 \pm 14.88$ & II \\
\hline 144 & 56.31813 & 32.10550 & 2.3 & -0.1 & 10.86 & 6.91 & $3999.79 \pm 13.69$ & II \\
\hline 145 & 56.32425 & 32.20164 & 0.0 & 0.0 & 1.59 & -1.00 & $3297.43 \pm 7.36$ & II \\
\hline 146 & 56.33525 & 32.10958 & 3.0 & -1.1 & 12.22 & 11.76 & $3948.52 \pm 5.03$ & II \\
\hline 147 & 56.34225 & 32.09586 & 0.0 & 0.0 & 4.86 & -1.00 & $-1.00 \pm-1.00$ & II \\
\hline 148 & 56.35454 & 32.22125 & 0.0 & 0.0 & 6.72 & -1.00 & $-1.00 \pm-1.00$ & II \\
\hline 149 & 56.35479 & 32.15842 & 5.1 & -1.3 & 2.40 & 4.14 & $3334.15 \pm 3.73$ & II \\
\hline 150 & 56.39846 & 31.99847 & 0.0 & 0.0 & 2.01 & -1.00 & $3261.67 \pm 8.00$ & II \\
\hline 152 & 55.93588 & 32.13828 & 0.6 & -1.0 & 9.30 & 7.22 & $4010.30 \pm 11.14$ & $\mathrm{II}^{*}$ \\
\hline 153 & 56.02829 & 32.13169 & 0.6 & 0.3 & 11.63 & 17.47 & $3357.78 \pm 13.27$ & $\mathrm{II}^{*}$ \\
\hline 154 & 56.03133 & 32.06914 & 0.0 & 0.0 & 2.49 & -1.00 & $3114.84 \pm 22.35$ & $\mathrm{II}^{*}$ \\
\hline 155 & 56.09408 & 32.03158 & 0.0 & 0.0 & 1.86 & -1.00 & $3788.72 \pm 5.01$ & $\mathrm{II}^{*}$ \\
\hline 156 & 56.10646 & 32.10478 & 0.0 & 0.0 & 10.98 & -1.00 & $3829.15 \pm 11.38$ & $\mathrm{II}^{*}$ \\
\hline 157 & 56.15783 & 32.13450 & 1.7 & -0.5 & 10.27 & 6.91 & $3890.10 \pm 11.85$ & $\mathrm{II}^{*}$ \\
\hline 158 & 56.17387 & 32.20064 & 0.0 & 0.0 & 4.92 & -1.00 & $3300.23 \pm 12.39$ & $\mathrm{II}^{*}$ \\
\hline 159 & 56.24046 & 32.12825 & 0.0 & 0.0 & 3.90 & -1.00 & $-1.00 \pm-1.00$ & $\mathrm{II}^{*}$ \\
\hline
\end{tabular}

\subsubsection{Literature mm detections}

Recently, Ruíz-Rodríguez et al. (2018) published an ALMA study of the discs in the IC 348 region, undertaken at $1.3 \mathrm{~mm}$. This study performed a snapshot survey of 136 sources classified as Class II objects by Lada et al. (2006) and Muench et al. (2007), detecting 40 with a $3 \sigma$ sensitivity of $\sim 0.45 \mathrm{mJy}$ at $1.3 \mathrm{~mm}$. This noise limit is roughly two times lower than the best noise floor the LMT achieves at $1.1 \mathrm{~mm}$ in this study; however, as we discuss below in Section 3.3, the expected scaling of the flux with wavelength makes the LMT 
Table 3. Table of LMT data sources (cf. Table 2) matched to the source list of Gutermuth et al. (2009).

\begin{tabular}{lc}
\hline Designation & Number \\
\hline \multicolumn{1}{c}{ LMT + Gutermuth et al. (2009) } \\
Matches & 116 \\
Detections & 28 \\
Upper limits & 88
\end{tabular}

Ruíz-Rodríguez et al. (2018) + Gutermuth et al. (2009)

$\begin{array}{lc}\text { Matches } & 116 \\ \text { Detections } & 40 \\ \text { Upper limits } & 76\end{array}$

Cottaar et al. (2014) + Gutermuth et al. (2009)

$\begin{array}{lc}\text { Matches } & 100 \\ \text { LMT detections } & 24 \\ \text { LMT upper limits } & 61 \\ \text { Matches not covered by LMT } & 15\end{array}$

competitive in placing physical constraints on the systems. Of the 136 sources classified as Class II objects by Lada et al. (2006) and Muench et al. (2007), 20 are not classified as Class II objects in the catalogue of Gutermuth et al. (2009). ${ }^{1}$ We exclude the 20 sources from Lada et al. (2006) and Muench et al. (2007) that are not included in Gutermuth et al. (2009) from our analysis of Class II sources.

Additional previous studies have targeted IC 348 at $\mathrm{mm}$ wavelengths. Lee et al. (2011) surveyed 85 sources in IC 348 with the Submillimeter Array (SMA) at $1.3 \mathrm{~mm}(230.5 \mathrm{GHz})$ and detected 10 sources with fluxes greater than $2 \mathrm{mJy}$. Espaillat et al. (2012) observed five sources in IC 348 with the SMA at $0.86 \mathrm{~mm}$ $(345 \mathrm{GHz})$, obtaining two detections with fluxes greater than 15 mJy. Cieza et al. (2015) detected 13 sources at $850 \mu \mathrm{m}$ with fluxes greater than $5 \mathrm{mJy}$. Owing to the small sample sizes of these studies (particularly compared to Ruíz-Rodríguez et al. 2018) and the uncertainty relating to translating fluxes between wavelengths (see Section 3.3), we opt to use only the observations of Ruíz-Rodríguez et al. (2018) in our analysis.

\subsubsection{Stellar properties}

For stellar characterization, we use previous studies to determine spectral type, effective temperature, bolometric luminosity, and mass of the stars hosting circumstellar discs. IC 348 was observed by the ancillary program INfrared Spectra of Young Nebulous Clusters (IN-SYNC; Cottaar et al. 2014), part of the Apache Point Observatory Galactic Evolution Experiment (APOGEE; Zasowski et al. 2013; from the third Sloan Digital Sky Survey, Eisenstein et al. 2011). IN-SYNC uses a multi-object, high-resolution $(R \sim 22500)$ spectrograph with a spectral coverage from 1.51 to $1.69 \mu \mathrm{m}$. In Cottaar et al. (2014), stellar parameters are derived by matching $H$ band spectra to a grid of synthetic spectra using a $\chi^{2}$ minimization technique and Monte Carlo Markov Chain technique to estimate errors. The fits have five parameters: effective temperature, surface gravity, radial velocity, rotational velocity, and $H$-band veiling

\footnotetext{
${ }^{1}$ The unified list of Class II objects between Lada et al. (2006), Muench et al. (2007), and Gutermuth et al. (2009) has 116 members, coincidentally the same number of sources we observed from the Gutermuth et al. (2009) sample alone.
}

(to account for emission from a hot inner circumstellar disc). Allowing physical parameters to vary allows for a more robust characterization of the stellar source than mapping spectral types to temperatures, particularly for young objects (see Bary \& Petersen 2014 for an example).

The source list of Gutermuth et al. (2009) was matched to the source list of IN-SYNC (Cottaar et al. 2014) at a tolerance of $1.0 \mathrm{arcsec}$, returning 100 matches. The remaining 60 sources from Gutermuth et al. (2009) were not observed, principally owing to the limit magnitude of IN-SYNC in $H$-band (typically $H<12.5$ ). The list of 100 matched sources was matched with a maximum radial tolerance of 5 arcsec to the flux-weighted centroid from the LMT source list. The middle frame of Table 3 lists the summarized matching numbers between the Gutermuth et al. (2009) and INSYNC, with additional unification with the LMT-measured sources. In principle, it is possible to use the same BT-SETTL model isochrones (Allard, Homeier \& Freytag 2011) as IN-SYNC to determine the luminosity, mass, and age of stellar sources. The luminosity, mass, and age are determined by a simple one-to-one mapping to temperature and surface gravity similar to the procedure used in Andrews et al. (2013); we interpolate a grid of effective temperature and surface gravity from the BT-SETTL models to determine luminosity, mass, and age of stellar sources. However, owing to the uncertainties that are introduced by the models, we choose to restrict ourselves to the published stellar parameter values and do not include the uncharacterized 60 sources in our discussion regarding the relationship of stellar parameters and disc flux.

Additionally, using IN-SYNC data, Fernandez et al. (2017) found two of the disc-bearing sources from Gutermuth et al. (2009) to be binaries (Sources 116 and 158, using the identification of Gutermuth et al. 2009 and Table 2). Both were covered by our sample, but were not detected. Upper limits of 2.1 and $4.92 \mathrm{mJy}$ were measured, respectively.

\subsection{Source matching}

We use the source list of Gutermuth et al. (2009) as the master list of YSOs in IC 348, a total of 160 sources. In Table 3, we summarize the matching number of sources between Gutermuth et al. (2009), the LMT detections from this study (discussed in Section 2.2), the stellar properties of Cottaar et al. (2014), and the ALMA study of Ruíz-Rodríguez et al. (2018). The middle frame of Table 3 lists the summarized matching numbers between the Gutermuth et al. (2009) and Ruíz-Rodríguez et al. (2018).

There are 141 YSOs (139 from Gutermuth et al. 2009, with two additional candidates discussed below in Section 3.2) that lie within the ALMA and LMT footprints, with each telescope covering 116 sources (source overlap means that the samples are not distinct, despite both samples coincidentally having 116 sources from the list of Gutermuth et al. 2009). Of those sources covered, the LMT detects 28 and ALMA detects 40, with 24 sources in common between the two samples. As a result, we have 44 sources with $\mathrm{mm}$ flux measurements from one of the two programs; choosing the more stringent upper limit measurement for the remainder results in a sample of 97 upper limits, with 74 from ALMA and 23 from LMT. When the more stringent limit is chosen, the LMT provides coverage of 51 sources, while ALMA provides coverage of 90 sources. The comparison is summarized in Table 4 .

Additionally, we queried Gaia Data Release 2 (Gaia Collaboration 2016, 2018) in a 30 arcmin radius circle for Class II sources in IC 348, as determined by Gutermuth et al. (2009). Using a matching tolerance of $1 \mathrm{arcsec}$, we find 127 of the 160 sources to 
Table 4. Table of ALMA sources matched to the LMT photometry. We exclude the 20 ALMA targets that are not infrared excess sources from Gutermuth et al. (2009) for this matching. We also exclude two candidate TDs that do not appear in the Gutermuth et al. (2009) list for this matching.

\begin{tabular}{ll}
\hline Designation & Number covered
\end{tabular}

Total Gutermuth et al. (2009) coverage (LMT-ALMA union)

Total LMT-ALMA overlap (LMT-ALMA intersection)

139

Overlapping detections

LMT limits, ALMA detections

ALMA limits, LMT detections

ALMA limits, deeper than LMT limits

LMT detections, no ALMA coverage

ALMA detections, no LMT coverage

ALMA limits, no LMT coverage

LMT limits, no ALMA coverage

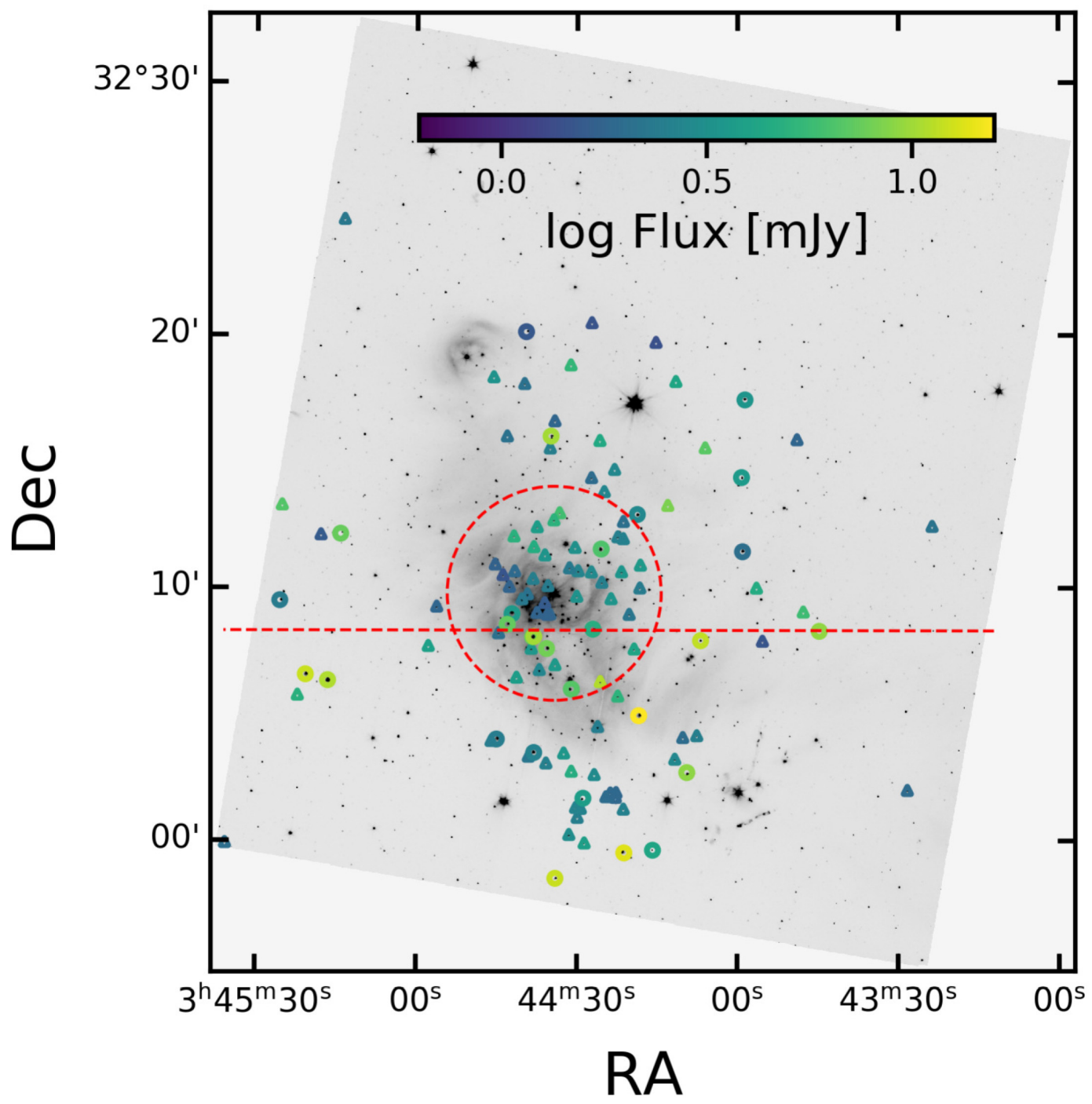

Figure 2. IRAC Channel $2(4.5 \mu \mathrm{m})$ greyscale image of the IC 348 region. The spatial distribution of YSOs measured as part of the survey, drawn from the sample of Gutermuth et al. (2009) is overlaid, with circle symbols representing discs from the LMT sample, and triangles representing upper limits. The symbols are colour-coded to correspond to the flux in mJy. Two partitions, separating the equal populations, are shown. An inner/exterior partition based on the central B5 binary is shown as a red dashed circle. A north/south partition (red dashed line) is also shown. 

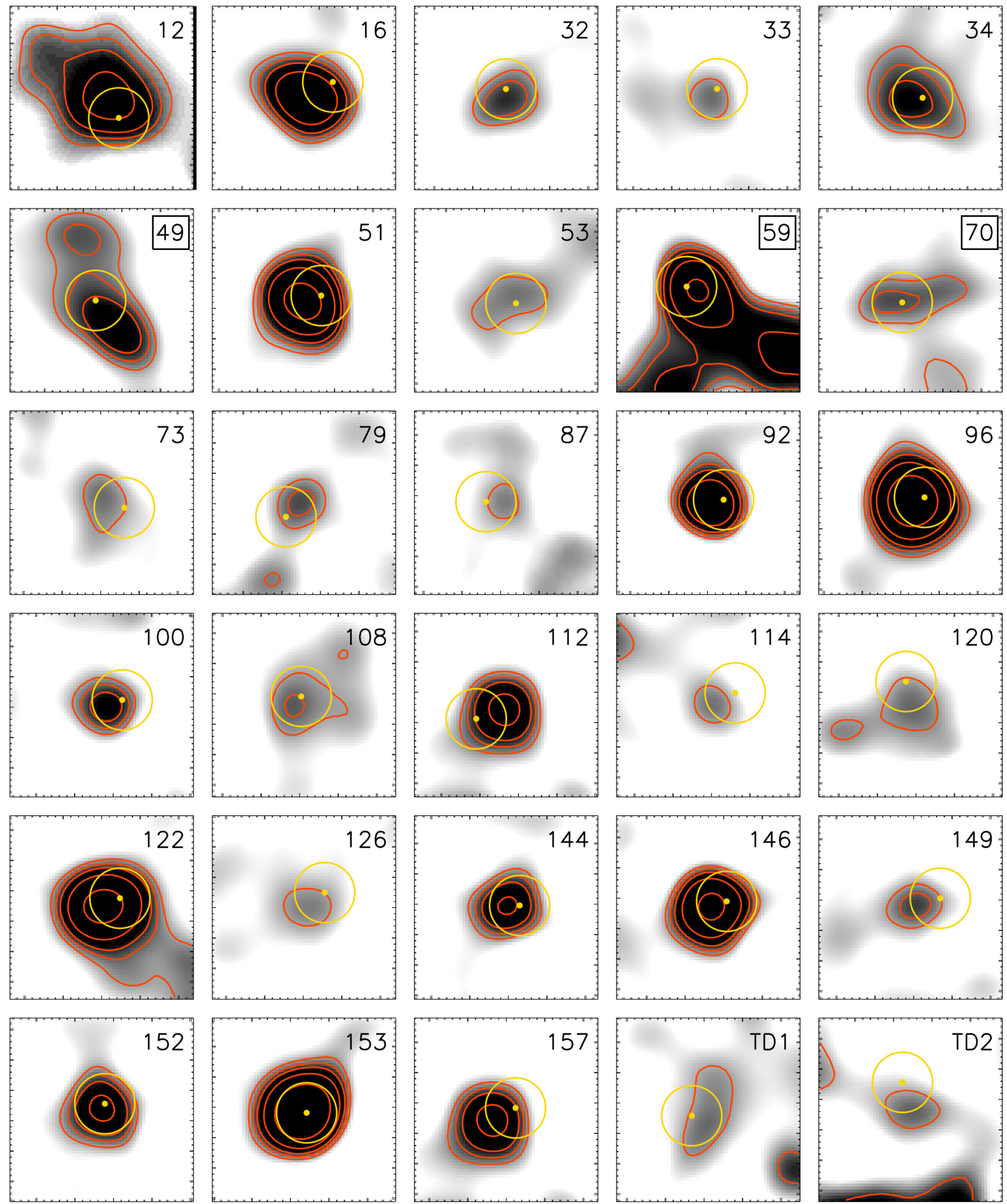

Figure 3. Inverse greyscale cropped LMT/AzTEC $1.1 \mathrm{~mm}$ images of the $30 \operatorname{arcsec} \times 30$ arcsec surrounding IC $348 \mathrm{members} \mathrm{detected} \mathrm{at} 1.1 \mathrm{~mm}$. The numbers in the upper right corner of each panel correspond to the Gutermuth et al. (2009) identifiers, as in Table 2. The panels labelled 'TD1' and 'TD2' correspond to the candidate TDs, as in Table 5. The greyscale stretch is linear, with white to black spanning from -1 to 5 times the median noise value for each field of view. The red contours mark $\mathrm{S} / \mathrm{N}=2.5,3.5,4.5,6.5,10.5,18.5$. The gold plot points mark the location of each matched YSO, and the surrounding circle shows the 5 arcsec matching radius. Objects 49,59 , and 70 are marked to draw attention to their substantial confusion with adjacent cloud material, and are treated as upper limits in our statistical analysis. 


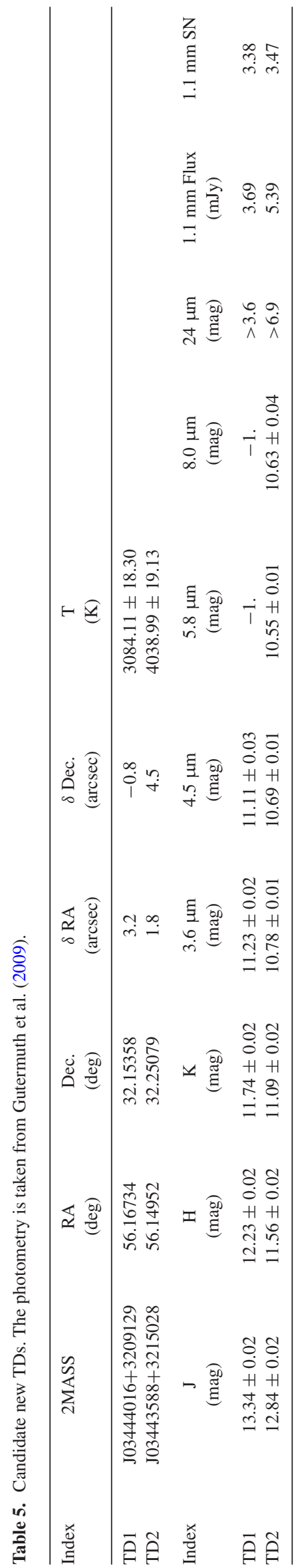

have reliable matches. Of the 127 sources, 99 sources have parallax measurements, with a median distance of $322.6 \pm 28.5$ pc. We therefore adopt $320 \mathrm{pc}$ value as the distance to the cluster, in line with previous estimates and other works. Of the 20 sources observed in Ruíz-Rodríguez et al. (2018) that do not appear in the IR excess list of Gutermuth et al. (2009), seven are present in Gaia DR2, and are all consistent with being at the distance of IC 348 (within $2 \sigma$ of $320 \mathrm{pc})$.

\section{RESULTS}

In this section, we first present the measurements from our LMT survey in Section 3.1. Section 3.2 reports two new candidate TDs serendipitously identified in our fields. In Section 3.3, we combine our results with that of Ruíz-Rodríguez et al. (2018) to make a master list of disc detections in IC 348, described in Section 3.4.

\subsection{LMT measurements at $1.1 \mathrm{~mm}$}

Table 2 lists the LMT data for 116 sources from Gutermuth et al. (2009), and Fig. 3 shows those YSOs with associated LMT/AzTEC detections. In Fig. 2, we plot the measured $1.1 \mathrm{~mm}$ fluxes as colourcoded symbols on top of the IRAC Channel 2. Detected sources are shown as circles, while upper limits are squares. As listed in Table 3, we detect 28 sources from the list of Gutermuth et al. (2009), with 88 additional upper limits. We detect $\frac{2}{4}=0.5$ of the Class I objects covered, and $\frac{26}{112}=0.232$ of known Class II objects covered (including TDs). For later analysis (Section 4.1), we show two methods with which to partition the data in Fig. 2, based on the most massive system of IC 348, the B5 binary HD 281159: a north/south cut on the declination of the B5 system, and an interior/exterior cut based on a 5 arcmin radius projection drawn from the B5 system. Both samples are divided such that the number of objects in each partition is equal.

As has been used extensively in the literature, a disc mass can be crudely estimated from a simple scaling of the mm-wave continuum luminosity (Hildebrand 1983; Beckwith et al. 1990). Assuming the emitting dust is optically thin and isothermal, the dust mass may be described as

$M_{\mathrm{d}}=\frac{F_{v} \cdot d^{2}}{\zeta \cdot \kappa_{v} \cdot B_{v}}$,

where $\kappa_{v}$ is dust opacity, $\zeta$ is the dust-to-gas mass ratio, $d$ is distance, and $B_{v}\left(\left\langle T_{\mathrm{d}}\right\rangle\right)$ is the Planck function at the average disc temperature. Following Andrews \& Williams (2005) and Andrews et al. (2013), we use $\zeta=0.01, \kappa_{v}=2.3 \mathrm{~cm}^{2} \mathrm{~g}^{-1}$. The uncertainty in $\zeta$ and $\kappa_{v}$ is likely to dominate, with errors up to an order of magnitude possible. We take $1.1 \mathrm{~mm}$ to be the reference wavelength, and follow the standard assumption in Andrews \& Williams (2005) that $\left\langle T_{\mathrm{d}}\right\rangle \simeq 20 \mathrm{~K}$ is applicable for all discs, though we acknowledge that this assumption is also highly uncertain. Andrews et al. (2013) did use a relationship for host star luminosity and dust temperature, $\left\langle T_{\mathrm{d}}\right\rangle=25\left(L / L_{\odot}\right)^{1 / 4} \mathrm{~K}$ derived from a radiative transfer analysis including a disc which is not completely optically thin (such that there is an inner heating contribution), but owing to the small temperature range of the host stars in our sample (the majority satisfy $3.4<\log T_{\text {eff }}<3.8$ ) and the shallow slope of the relation, the dust temperature difference is trivial. As it is highly uncertain whether in the relationship between mm flux and $M_{\mathrm{d}}$ is a simple linear scaling, for lack of a clear answer, we will assume for our analysis that the mm flux scales monotonically with the dust mass in the disc, such that discs with higher flux are more massive. 
Further, mass determinations from models such as Espaillat et al. (2011) are consistently higher than Andrews \& Williams (2005). The opacities used are approximately three times lower and the models use a larger outer disc radius and self-consistent surface density and temperature instead of power-law approximations. Therefore, masses obtained by different models cannot be meaningfully compared. We reserve the modelling of disc SEDs for future work, particularly when the dust emissivity may be more precisely constrained.

For posterity, under the standard assumptions discussed above, we report that $F_{1.1 \mathrm{~mm}}=6.94 \mathrm{M}_{\oplus} / \mathrm{mJy}$, but do not use the disc masses in our analysis, noting instead that the disc mass under these assumptions is directly proportional to flux, and instead choose to perform our analysis on flux only.

As we discuss below, our data suggest a different spectrum for dust opacity, which translates into a different flux-to-mass conversion. Dust opacity in circumstellar discs is discussed extensively in Andrews \& Williams (2007), and we follow their equation (3) to provide a possible scaling $\kappa_{v}$ from $1.3 \mathrm{~mm}(225 \mathrm{GHz})$ to $1.1 \mathrm{~mm}$ (265.9 GHz)

$\kappa_{v}=\kappa_{0}\left(\frac{v}{v_{0}}\right)^{\beta}=3.55$.

Comparing with the typically assumed $\beta$ value, our implied $\beta$ will result in a factor of 1.55 change for the conversion of flux to mass. Therefore, a scaling of flux to mass with $F_{1.1 \mathrm{~mm}}=4.5 \mathrm{M}_{\text {earth }} / \mathrm{mJy}$ is also allowed by our data. As we will consistently refer to flux only throughout the paper, this range of mass conversions does not affect our results.

\subsection{New candidate transition discs discovered via mm-wave photometry}

The mm survey reported here targeted a list of known infrared excess sources from Gutermuth et al. (2009), including the 10 known TDs in IC 348. In addition, two known members of IC 348 (Cottaar et al. 2014) that were considered discless based on Spitzer IR observations were detected serendipitously in our $\mathrm{mm}$ maps, although at low S/N (see Fig. 3). We propose that these may be TDs with massive, cold outer discs and substantial inner gaps. Statistical comparisons to the more numerous lists of field stars in the Spitzer IR catalogue show that the small matching tolerance ( 5 arcsec) afforded by the excellent astrometry of the LMT means that the $\mathrm{mm}$ detections have a vanishingly small likelihood of being accidental matches to coincident IC 348 members.

Neither of the two newly identified candidate TDs was detected in Spitzer/MIPS $24 \mu \mathrm{m}$ coverage. To estimate the $24 \mu \mathrm{m}$ upper limits from the data, we computed a new, spatially resolved (30 arcsec), 90 per cent differential completeness map for the Gutermuth et al. (2009) MIPS source extraction, using the method of Gutermuth \& Heyer (2015). We plot the SEDs of the two candidates in Fig. 4, with the local $24 \mu \mathrm{m}$ completeness limits for each source shown in blue to demonstrate that the $24 \mu \mathrm{m}$ constraints cannot rule out excess emission. The objects appear to have strong $\mathrm{mm}$ emission consistent with a cold circumstellar disc. If further observations confirm their nature as TDs, it is suggestive that sensitive mm-wave continuum mapping of nearby star-forming regions, with facilities such as LMT and the new TolTEC continuum array camera (Bryan et al. 2018), are likely to discover more such inner-gap discs among stars where MIPS $24 \mu \mathrm{m}$ photometry simply could not constrain sufficiently to ensure that no disc is present.

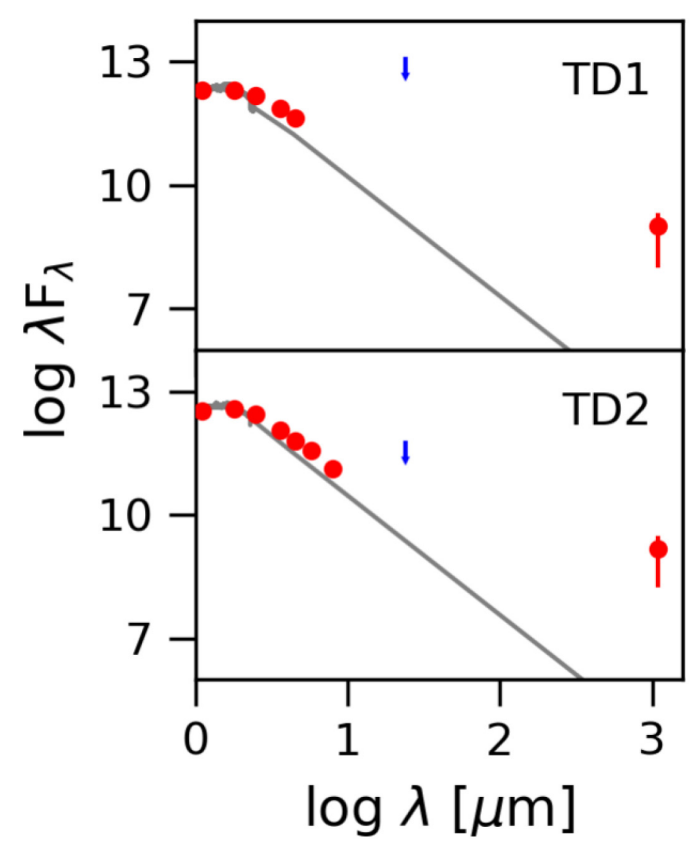

Figure 4. Spectral energy distributions of two candidate TDs. The grey spectra are an M5 (upper panel) and K7 (lower panel) from the spectral library of Rayner, Cushing \& Vacca (2009), extrapolated using a RayleighJeans approximation beyond $5 \mu \mathrm{m}$. The choice of comparison spectrum corresponds to the effective temperature measured from IN-SYNC data. For both, the measured $24 \mu \mathrm{m}$ point is an upper limit (shown in blue). Owing to the non-constraining nature of these limits, combined with the lack of significant excess emission in the mid-infrared, the sources were classified as discless by Gutermuth et al. (2009).

The possibility that still-undiscovered TDs lurk in nearby starforming regions sheds new light on the apparent disagreement between IC 348 and other young clusters put forth in Muzerolle et al. (2010), which found that 12 per cent of the discs in IC 348 are TDs. Additionally, the discovery of new TDs via a deep mm search provides an interesting avenue for identifying systems where planets may be forming. As we cannot be certain that our sample is complete to unidentified TDs, we do not place a constraint on the fraction of TDs, but rather advocate for a survey that includes all known members whose MIPS $24 \mu \mathrm{m}$ flux cannot rule out the presence of a TD. Further, other regions may benefit from blind searches, particularly as new facilities that can perform such obervations come online.

\subsection{Connection with ALMA}

Given the opportunity to compare data sets, we wish to examine our results as compared to those of ALMA to characterize the sources. Fig. 5 plots LMT and ALMA fluxes for matched sources (i.e. the 24 mutual detections). We additionally show 11 sources with ALMA detections but no LMT detection (grey arrows), as well as two sources with LMT detections and no ALMA detection (blue arrows). With the 24 overlapping sources, the mean of the ratio for detected sources is 0.476 (unweighted) and 0.461 (flux weighted), as indicated by the dashed line.

The conversion of data points to different wavelengths relies on the relationship $F_{v} \propto \nu^{\alpha}$, where $\alpha=\beta+2$. $\beta$, the dust emissivity, 


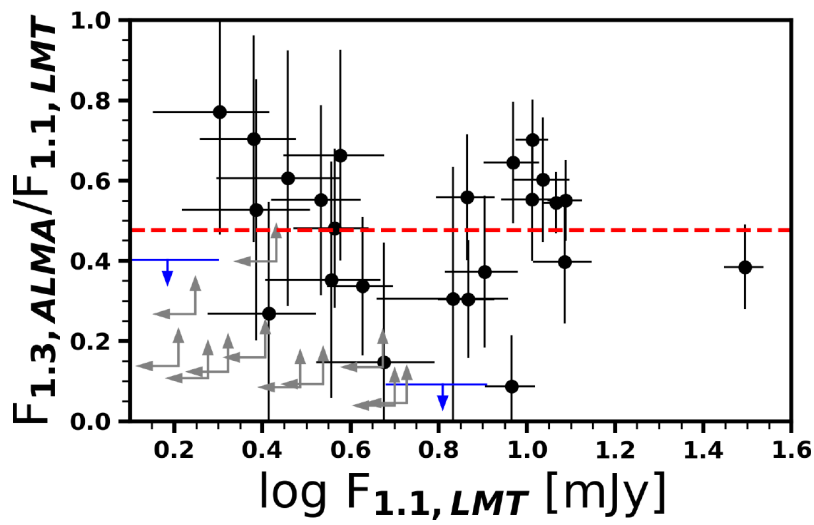

Figure 5. Ratio of ALMA $1.3 \mathrm{~mm}$ fluxes to LMT $1.1 \mathrm{~mm}$ fluxes as a function of LMT fluxes for 24 matched sources. The black points have detections in both the ALMA and LMT surveys. The grey arrows have detections in ALMA but upper limits in the LMT data, and are thus lower limits. The blue arrows have detections in the LMT data but no detection in ALMA, and are therefore upper limits.

is related to the flux ratio by

$$
\begin{aligned}
\beta & =\frac{\log \left(F_{1.3 \mathrm{~mm}} / F_{1.1 \mathrm{~mm}}\right)}{\log (1.1 / 1.3)}-2 \\
& =-13.7 \log \left(\frac{F_{1.3 \mathrm{~mm}}}{F_{1.1 \mathrm{~mm}}}\right)-2 .
\end{aligned}
$$

The dust emissivity is related to the dust opacity as $\kappa_{v}=C v^{\beta}$, where $C$ is some constant. The theoretically expected value at long wavelengths, via the Kramers-Kroenig relations (Purcell 1969), is $\beta=2$.

Assuming 15 per cent uncertainty in each mean flux, ${ }^{2}$ the uncertainty in the mean flux ratio is $\sim 21$ per cent, and thus the uncertainty in the logarithm of the ratio is 0.09 . Our flux-weighted finding of $F_{1.3 \mathrm{~mm}} / F_{1.1 \mathrm{~mm}}=0.461$ propagates to $\beta=2.61 \pm 1.24$. As we report a $1 \sigma$ error bar, we have no strong constraint on $\beta$ here, but we are consistent with theoretical predictions. Unfortunately, we are severely limited by the independent calibration uncertainties and the narrow wavelength difference. However, in the Taurus region, Andrews et al. (2013) found $\alpha=2.4 \pm 0.5$, which is equivalent to $\beta=0.4$, a stark departure from what we calculate here, although both measurements are statistically consistent owing to their large uncertainties. The strongly different values of $\beta$ again caution against simple scaling of mm flux to disc mass. The uncertainty in the LMT photometry makes drawing detailed conclusions about the SED slope difficult at present for individual sources, but future instruments will be able to provide more information. Throughout the rest of the work, we will use the relationship $F_{1.1 \mathrm{~mm}}=2.12 F_{1.3 \mathrm{~mm}}$ to transform IC 348 data from Ruíz-Rodríguez et al. (2018), as calibrated above.

\subsection{Merged disc list}

We use the infrared excess list of Gutermuth et al. (2009) as our guide to create a master list of disc measurements in IC 348. Of the 136 sources reported in Ruíz-Rodríguez et al. (2018), 20 do not appear on the list in Gutermuth et al. (2009), indicating that they

\footnotetext{
${ }^{2}$ We have an error of a few percent on the individual fluxes, but we
} acknowledge a likely 10 per cent overall calibration uncertainty. may have been misclassified as disc-bearing objects in previous studies (Lada et al. 2006; Muench et al. 2007). For the purposes of our research here, we exclude those sources from the candidate disc sample. Doing so does not exclude any of the detections from Ruíz-Rodríguez et al. (2018), only upper limits. As none of the 20 sources in the sample of Ruíz-Rodríguez et al. (2018) without a match in the Gutermuth et al. (2009) catalogue have detections, we interpret this as supporting evidence that the sources were likely misclassified.

As discussed in Section 2.4 and summarized in Table 4, we compile a unified source list which covers 139/160 infrared excess sources identified in Gutermuth et al. (2009), including 93/139 with temperature measurements from IN-SYNC (Cottaar et al. 2014). With the addition of the two candidate TDs, we create a master list of mm flux measurements for 141 sources with infrared (or potential infrared) excess. We choose to compile the fluxes at $1.1 \mathrm{~mm}$. Therefore, we prioritize LMT detections in cases with overlap (the 28 LMT-detected sources, cf. Table 3 ) as the primary detections, supplementing with 16 ALMA scaled detections. For limits, we first scale the ALMA measured upper limits, then select the more stringent limit in cases of overlap. This sample is analysed in Section 4.1. Comparing this net sample to IN-SYNC, we find that 25/28 LMT detections have stellar parameters, as well as 12/16 ALMA detections. 11/23 LMT upper limits and 46/74 ALMA upper limits have stellar parameters. Summing the numbers, 94/141 sources have stellar parameters. The cross-matched sample is used in Sections 4.2 and 4.3.

Unfortunately, owing to the incomplete nature of the observations of the Class II sample from Gutermuth et al. (2009), as well as the possible presence of previously unknown TDs, we can only place a loose constraint on the $1.1 \mathrm{~mm}$ disc mass fraction with $f_{1.1 \mathrm{~mm}}>1 \mathrm{mJy}: f_{\mathrm{d},>1 \mathrm{mJy}}=\frac{44}{141}=0.31_{-0.04}^{+0.09}$. The upper (lower) limit is calculated assuming all 21 sources in the Class II sample of Gutermuth et al. (2009) for which we do not have coverage are measured to have fluxes above (below) $1 \mathrm{mJy}^{3}$ This is similar to the number reported in Ruíz-Rodríguez et al. (2018): $f_{\mathrm{d},>1 \mathrm{mJy}}=$ $\frac{40}{116}=0.34$, when only counting sources defined by Gutermuth et al. (2009).

\section{ANALYSIS}

In this section, we first present the luminosity function of the detected discs in IC 348 in Section 4.1. We present basic analysis of the millimetre measurements as related to additional information on the stellar hosts (Section 4.2), including rotation rates (Section 4.3).

\subsection{Cumulative luminosity function}

Examining Fig. 2, we do not see any obvious spatial correlations with our detections, including a lack of correlation with the infrared nebulosity. However, the lower half of the cluster contains almost all of the bright detections $\left(\log F_{\mathrm{mm}}>0.6\right)$, consistent with a south-to-north age gradient in the cluster. Additionally, the centre of the cluster, within 5 arcmin of the central B5 binary, appears to have a dearth of disc detections. In this section, we will use a product-limit estimator formalism and cumulative luminosity functions to show that the north/south populations are distinct at

\footnotetext{
${ }^{3}$ If we were to include the 20 potentially misclassified sources observed in the sample of Ruíz-Rodríguez et al. (2018), the disc fraction would reduce to $f_{\mathrm{d},>1 \mathrm{mJy}}=\frac{44}{161}=0.27_{-0.03}^{+0.09}$.
} 


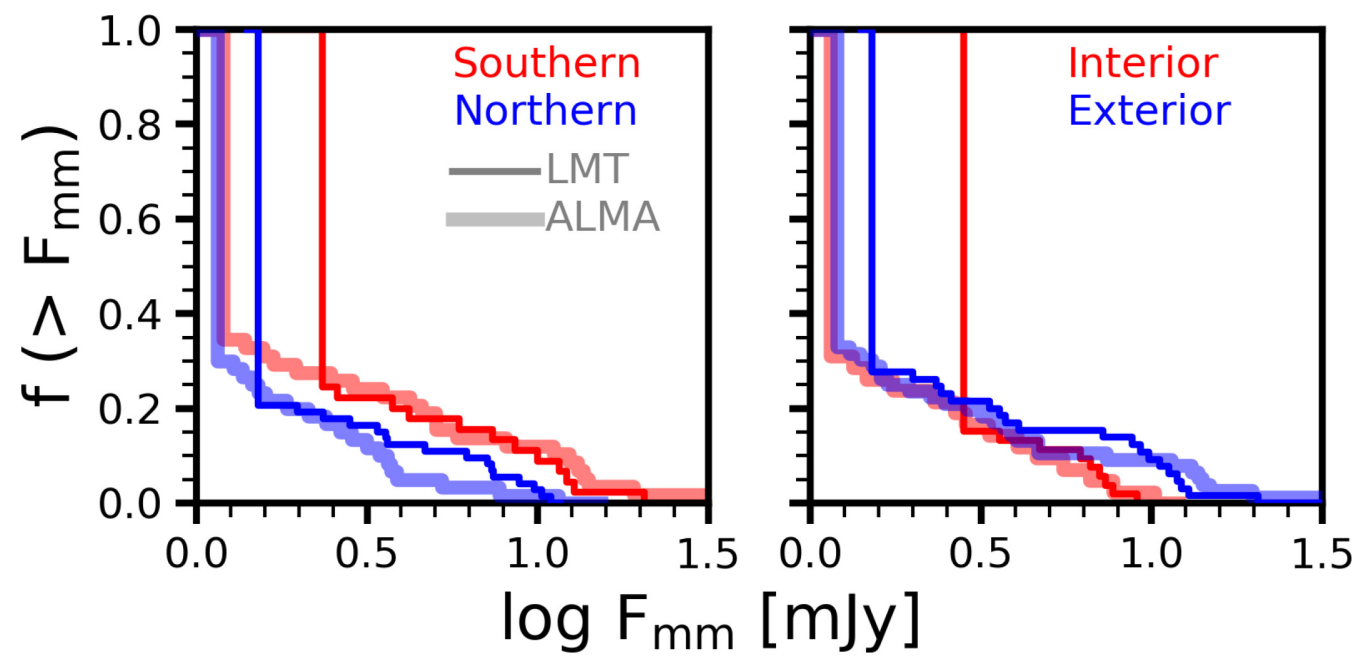

Figure 6. Cumulative flux distributions for IC 348. Left-hand panel shows subdivision of northern and southern halves, as indicated in 2, right-hand panel shows subdivision of cluster interior ( $<5$ arcmin from the central B5 binary) and cluster exterior, also indicated in 2 . The solid lines are for the LMT results only, transparent thick lines are for ALMA results only. ALMA sources have been scaled to be at $1.1 \mathrm{~mm}$.

high significance, and that the interior/exterior populations are also distinct, albeit at a more modest significance. We interpret this as evidence for (1) asynchronous star formation across the cluster, and/or (2) environmental effects from the central B5 binary.

Fig. 6 presents the cumulative $1.1 \mathrm{~mm}$ luminosity distribution functions for IC 348, divided into north/south and interior/exterior partitions (left- and right-hand panels, respectively). The distributions are made following the formalism for Kaplan-Meier productlimit estimator to properly account for censored measurements (upper limits on $F_{\mathrm{mm}}$ ) as described in Feigelson \& Nelson (1985). In such a formalism, the high upper limits of some LMT sources can lead to a truncated distribution, owing to the uncertainty of the disc distribution function structure below the limit. Therefore, we also perform the same analysis on the ALMA results of RuízRodríguez et al. (2018), finding indistinguishable curves, except for the northern partition, which is missing three relatively high-flux sources in Ruíz-Rodríguez et al. (2018).

The primary metric for comparing two censored samples used in the literature is the Peto-Prentice test (Feigelson \& Nelson 1985). The test returns $p_{\emptyset}$, the probability that two samples are drawn from the same parent distribution. As with many other statistical measures, the power of the metric lies in the ability to confidently say that distributions are not drawn from a parent distribution. Unlike previous mm disc detection works (e.g. Andrews et al. 2013; RuízRodríguez et al. 2018), we do not construct a cumulative distribution of $p_{\emptyset}$, the so-called null hypothesis probability distribution $f\left(<p_{\emptyset}\right)$. The purpose in those works was to homogenize the sample of spectral types between different star-forming regions; in the case of IC 348, an examination of both the north/south and interior/exterior partitions does not show significant differences in the temperature distributions. Likewise, an examination of the spectral type distributions between the ALMA and LMT samples does not reveal any significant differences (compare the range of temperatures in Fig. 9).

Despite the apparent differences by eye in both panels of Fig. 6, the Peto-Prentice does not find strong evidence for differentiation between the two samples. The lack of sensitivity in the two-sample comparison is likely owing to the relatively small dynamic range of the detections. While the LMT north/south partition returns $p_{\emptyset}=0.029$, significant at the $\sim 2.5 \sigma$ level, the same test on the unified LMT-ALMA data set returns $p_{\emptyset}=0.33$, meaning the samples are indistinguishable given this test. ${ }^{4}$ The interior/exterior partitions place even less stringent constraints, with $p_{\emptyset}=0.21$ for the LMT only and $p_{\emptyset}=0.47$ for combined sample.

However, we can use the Peto-Prentice formalism to investigate the importance of the choice of flux scaling that transforms the ALMA points to the LMT reference wavelength: $F_{1.1 \mathrm{~mm}}=C F_{1.3 \mathrm{~mm}}$. In Section 3.3, we calculated $C=2.12$, but the $\alpha$ value of 2.4 derived by Andrews et al. (2013), using a sample of 60 sources in Taurus with multiple detections between 0.7 and $3 \mathrm{~mm}$, corresponds to $C=1.49 \pm 0.13$. The Andrews et al. (2013) value for $C$ has been used throughout the literature to homogenize data sets across different wavelengths for comparison between clusters (e.g. Ruíz-Rodríguez et al. 2018). We test the validity of $C_{\text {Taurus }}$ being used for IC 348 by comparing the cumulative distribution functions between the LMT and scaled ALMA distributions for a range of $a$ values. When we use $C=2.12$, we find $p_{\emptyset}=0.59$, well within $1 \sigma$, implying that the distributions cannot be distinguished. When using the $C_{\text {Taurus }}=1.49$, we find $p_{\emptyset}=0.13$, still within $2 \sigma$, and thus indistinguishable. At the $3 \sigma$ level, where it can be strongly stated that the samples are not drawn from the same parent distribution, we can exclude $C_{\text {IC } 348}<0.84$ and $C_{\text {IC } 348}>18.25$. While these limits are not particularly constraining, as $C<1$ is aphysical, at the $2 \sigma$ level, we can exclude $C_{\mathrm{IC} 348}<1.18$ and $C_{\mathrm{IC} 348}>7.81$. Fig. 7 shows the distribution of $\log p_{\emptyset}$ as a function of $C$. A more complete, deeper sample with multiple wavelength detections would be able to use this formalism to place more stringent limits.

In Fig. 8, we perform two-sample tests on the divided IC 348 samples, as a function of the number of flux-sorted discs included. Our goal is to calculate the difference between the populations of bright discs in the partitions. At each abscissa value $x$, we calculate the two-sample null probability for the north/south and interior/exterior population divisions. We select the $x$ brightest discs from each sample and compare the probability the populations

${ }^{4} \mathrm{We}$ assume the probabilities are drawn from a $\chi^{2}$ distribution, such that $p_{\emptyset}=0.317,0.0455,0.0027$ correspond to the nominal $1 \sigma, 2 \sigma$, and $3 \sigma$ probabilities. 


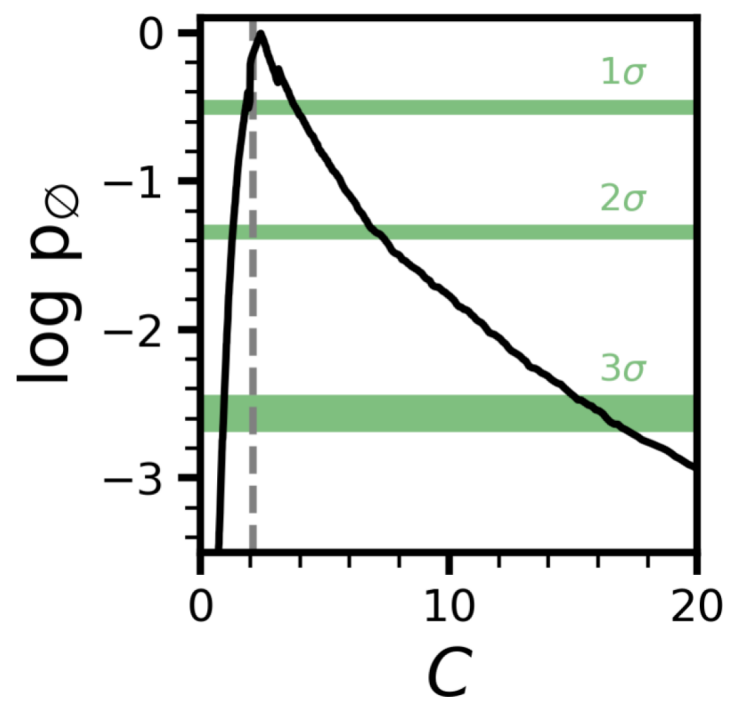

Figure 7. Log null probability, $p_{\emptyset}$, versus $C$, the scaling to convert between 1.1 and $1.3 \mathrm{~mm}$ fluxes: $F_{1.1 \mathrm{~mm}}=a F_{1.3 \mathrm{~mm}}$. The green horizontal lines denote $1 \sigma, 2 \sigma$, and $3 \sigma$ values, from top to bottom. The grey vertical dashed line is $a=2.12$, the nominal value assumed in this work.

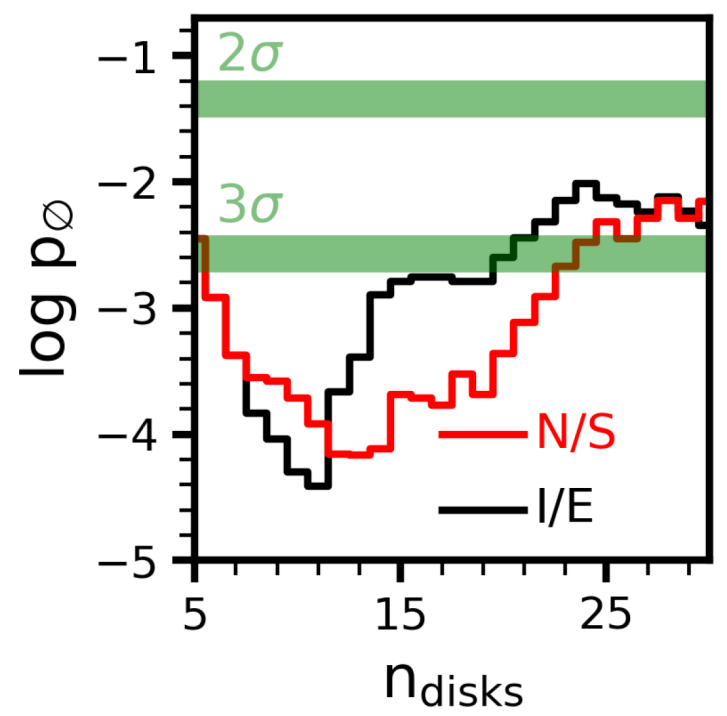

Figure 8. Log null probability, $p_{\emptyset}$, as a function of flux-sorted disc samples. At each value of $n_{\text {discs }}$, we perform a two-sample test on the $n$ brightest discs from each of the separated populations and plot the resulting value of $p_{\emptyset}$, for both the north/south (black) and interior/exterior partitions (red). The horizontal green bands correspond to $2 \sigma$ (upper) and $3 \sigma$ (lower).

were drawn from the same sample. As the populations have been divided in half, it is a fair comparison to choose the number of discs rather than the fraction of discs. For $n_{\text {discs }}<23$ (19) in the north/south (interior/exterior) divisions, we see strong evidence $(>3 \sigma)$ for different populations. As the number of discs included increases, the signal decreases. We interpret this as evidence for (a) a north-south gradient in star formation, and if we assume that the disc flux (mass) declines with time, the discs in the south are younger, and/or (b) disc photoevaporation from the central B5 binary that reduces the mass (flux) of the discs in the vicinity. In a disc photoevaporation scenario, we would be observing a loss of mass from the less bound portions of large-radius discs, resulting in a truncation that preferentially affects the biggest discs the most. Ansdell et al. (2017) found similar evidence in the form of a dearth of discs around the OB stars in $\sigma$ Ori. Future high spatial resolution observations of discs in IC 348 are needed to test this hypothesis.

An alternative scenario is that the north-south gradient is evidence that the IC 348 region is inhomogeneous, and that the observations reflect the variation in density rather than age. Cottaar et al. (2015) fit the distribution of IN-SYNC radial velocities with one and two Gaussians, finding that the addition of a second Gaussian significantly improved the fit. The velocity dispersion is consistent with supervirial conditions, implying that the current evolution of the cluster is dominated by stellar and gas massloss. Cottaar et al. (2015) propose a myriad of possibilities for the observations, including that IC 348 is contaminated by the Perseus OB2 association, that IC 348 recently underwent a gas expulsion or merger event that created a dispersing halo of stars around IC 348, that IC 348 has not yet relaxed to a cluster with radial velocities that may be represented by a single Gaussian, and lastly that the region may be the superposition of two subclusters which are converging along the line of sight. The Gaia data presented by Ortiz-León et al. (2018) suggest based on proper motion analysis that a halo of stars may be present around the cluster, but future data releases will be needed to fully characterize the region.

\subsection{Dependences on stellar hosts}

In Fig. 9, we plot surface temperature versus $1.1 \mathrm{~mm}$ flux for all sources in the sample with IN-SYNC stellar parameters (see Table 4). The points are colour-coded with the IN-SYNC-derived $\log (g)$ values. When not covered by the LMT, we use ALMA observations for corresponding sources, scaled using a flux ratio of $F_{1.1 \mathrm{~mm}}=2.12 F_{1.3 \mathrm{~mm}}$. Overlapping sources between ALMA and the LMT are shown using the LMT points if detected, or as the more stringent limit in the case of upper limits (cf. Table 3). The addition of the ALMA detections, scaled as described in Section 3.3 to match the wavelength of the LMT, do not change the conclusions drawn from the LMT data alone, but provide valuable additional data.

To further demonstrate the strong difference between northern and southern partitions of IC 348, we divide the sources into those in the northern partition (left) and southern partition (right). While other regions such as Taurus (complete at temperatures hotter than M8.5 Andrews et al. 2013) exhibit a relationship (see discussion below in Section 5.2), we do not find evidence for a conclusive relationship between stellar surface temperature and $1.1 \mathrm{~mm}$ flux. One possible explanation for the lack of relationship comes from Cottaar et al. (2014), who also demonstrated a spread in intrinsic stellar radii, indicative of an age spread where younger stars have had less time to contract towards the main sequence. Using similar methodology where $\log (g)$ is an age indicator, Yao et al. (2018) also found weak evidence for intra-cluster age variation. Our interpretation of differing star formation histories in the north and south partitions supports the explanation of Cottaar et al. (2014) and Yao et al. (2018).

We do not detect emission from two cool stars $\left(T_{\text {eff }} \sim 2000 \mathrm{~K}\right)$ identified by Cottaar et al. (2014). The upper limits are reported in Table 2. Measurements of mm flux around very cool sources may not be uncommon; Ward-Duong et al. (2018) measured $885 \mu \mathrm{m}$ flux densities for 24 Taurus sources with late M spectral types, and Ruíz-Rodríguez et al. (2018) detect mm emission from a 


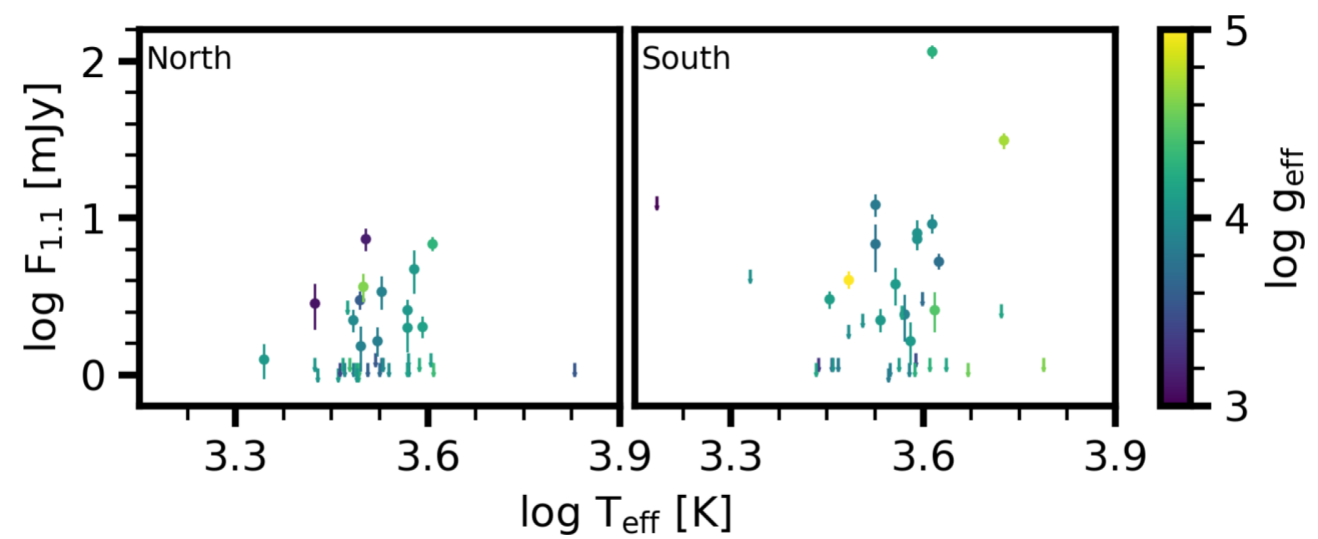

Figure 9. Flux versus effective temperature ( $\left.T_{\text {eff }}\right)$ for the discs with both measurements in our sample, colour-coded by $\log (g)$ values. The two panels have been divided for stars that are in the north division (left-hand panel) and south division (right-hand panel). The sources are drawn from the unified LMT and ALMA list, where the ALMA observations have been scaled (see the text).

simililar-temperature source, well below our detection threshold, and significantly below that of the detected source in our sample.

\subsection{Rotation rates}

The ancillary IN-SYNC data give an opportunity to analyse the influence of the outer disc on the rotation rates of the host star, using $v \sin i$ data. Rebull et al. (2006) found evidence in Orion to support inner, IR-excess discs limiting the spin-up of stars using a relationship between stellar periods, attributable to rotation, and IRAC [3.6] - [8.0], a common measure of dust in the inner disc. Cieza \& Baliber (2007) reported a similar relation in their study of Orion and NGC 2264. In contrast, Cieza \& Baliber (2006) did not find any evidence for a correlation between slow rotators and disc presence for IC 348. Herbst, Maley \& Williams (2000) did an early comparison of rotation periods for YSOs in Orion and IC 348, which we are in a position to update, finding very similar distributions for similar masses.

We make a comparison of rotation rate and disc presence between Orion and IC 348 using the data at hand. Herbst, Bailer-Jones \& Mundt (2001) found evidence for a decreasing median rotation period with increasing mass $0.1 \mathrm{M}_{\odot}<M<0.4 \mathrm{M}_{\odot}$. Thus, to perform a fair comparison between the $v \sin i$ distributions in IC 348 and Orion, one must have a similar distribution of masses. We use $T_{\text {eff }}$ as a proxy for mass, finding that for the stars observed by INSYNC, the distributions for IC 348 and Orion are similar, peaking at $\log \left(T_{\text {eff }}\right)=3.5$. The temperature spread is modestly more extended to lower and higher temperatures IC 348, but we are not aware of any bias this would introduce in the results.

We use the IRAC source list for Orion from Megeath et al. (2012) to match with the IN-SYNC study of Orion (Da Rio et al. 2016). In Fig. 10, we plot $v \sin i$ versus IRAC [3.6] - [8.0]. The histograms above and beside the main panel show the ratio of disc sources to total sources in bins of $\log v \sin i$ and [3.6] - [8.0], respectively. The background contours are a density estimate from the large sample available in Orion, showing two clear loci based on the presence or absence of a disc. Notably, the discless sources extend to larger values of $v \sin i$, supporting a conclusion that the presence of a disc acts to slow the rotation rate of a star, or at least limits the spin-up of stars to a similar maximum rotation rate.

IC 348 is shown as points in Fig. 10, partitioned first as discless sources (black) and YSOs from Gutermuth et al. (2009). For the
YSOs, we outline the points in cyan if we placed an upper limit, and blue if we detected the source. The grey circles with no outline were unobserved. The IC 348 sources have a similar distribution as those in Orion, including the extension of discless cluster members to higher $v \sin i$. The distribution and extent of colour for objects detected in $\mathrm{mm}$ and objects with limits in $\mathrm{mm}$ appear to be identical, based on Fig. 10. Performing a Kolmogorov-Smirnov test on the distribution of colours for $\mathrm{mm}$ detections and $\mathrm{mm}$ limits results in a $p$-value of 0.06 , suggesting that we cannot exclude the possibility that these samples came from the same distribution. Therefore, we argue that distribution of massive disc detections and non-detections in colour are indistinguishable.

This directly suggests that in IC 348 there is no correlation between disc mass and infrared excess indicating the presence of an inner disc. In the case of TDs, there may actually be an anticorrelation between disc mass and infrared excess as the inner disc wall is too cool to radiate at near-infrared wavelengths. At $\log v \sin i \sim 1.8$, a dearth of disc sources emphasize the extension of discless sources to higher $v \sin i$ in both regions (above $\log v \sin i=2$, the distribution of all sources falls off). In addition, the similarity to Orion suggests that any spin evolution of discless stars is largely confined to early evolution, as no relative spin evolution between the two regions is apparent, despite their differing isochronal ages. In principle, IC 348 would be expected to have higher $v \sin i$ relative to Orion in comparable mass ranges, owing to the spin-up of stars during evolution. However, we do not see evidence for faster spinning stars at the age of IC 348 , relative to Orion.

Though we see two clear loci hosting the disc-bearing and discless sources, a number of outliers are obvious in Fig. 10. Several of the outliers are near $\log v \sin i=3$, at least close to the break-up speed. We investigated the presence of binaries, and did not find any of the outlier sources to be binaries in the study of Fernandez et al. (2017). Orion has a similar number of sources that do not lie near the locus as the scattered point in IC 348.

\section{DISCUSSION}

In this section, we compare our result to the Taurus star formation region. In Section 5.1, we present evidence supporting the conclusion that the variation between clusters is confined to the brightest (most massive) discs. In Section 5.2, we then compare the stellar 


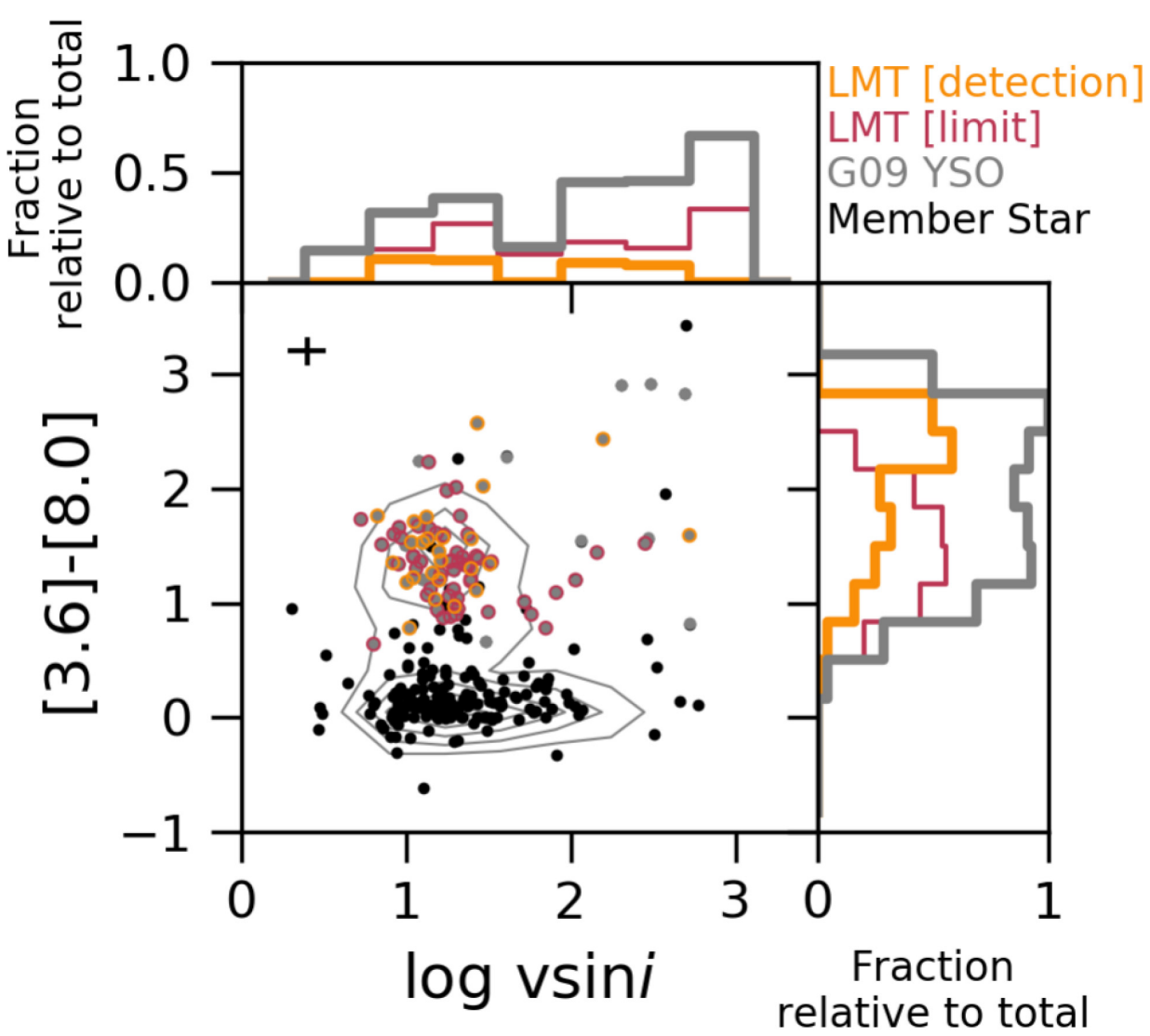

Figure 10. Main panel: $\log v \sin i$ versus [3.6] - [8.0], a common measure of disc presence, for IC 348 (points) and Orion (contours). Upper and right-hand panels show histograms with the fraction of disc sources to all sources divided by $v \sin i$ and [3.6] - [8.0], respectively. The cross in the upper left of the central panel indicates the median error bars on $v \sin i$ and [3.6] - [8.0].

surface temperature to millimetre luminosity between IC 348 and Taurus in detail, discussing the similarities and differences.

\subsection{High-mass disc evolution}

As discussed in the previous section, Taurus and IC 348 appear to contain a similar population of discs below a certain disc mass threshold. We extend this comparison to other nearby star-forming regions, using the disc fluxes reported for Lupus (Ansdell et al. 2016), Chamaeleon I (Pascucci et al. 2016), $\sigma$ Ori (Ansdell et al. 2017), and Upper Scorpius (Barenfeld et al. 2016). In order to homogenize the samples to match our IC 348 survey, we translate the flux using $F_{\lambda}=F_{1.1 \mathrm{~mm}} \times(1.1 \mathrm{~mm} / \lambda)^{\alpha}$, where we opt to set $\alpha=2.4$, following the slope determined by Andrews et al. (2013).

In Fig. 11, we show the cumulative disc function for all discs in each population (left-hand panel), and for all discs with $F_{1.1 \mathrm{~mm}}<$ $15 \mathrm{mJy}$ (right-hand panel). All sources below the dashed line are included in the higher flux analysis. The left-hand panel of Fig. 11 reproduces previous analyses, finding that the disc populations are appreciably different between regions, appearing to be roughly correlated with the age of clusters. However, in the right-hand panel, when we exclude the high-flux discs from the analysis, we find that the populations become consistent with one another, with the exception of the significantly older Upper Sco. In Table 6, we report the results of two-sample tests comparing IC 348 to the reference sample of each different population. The values show that while the total distributions disagree, the differences are confined to the highest mass discs (in that their elimination results in populations that are indistinguishable as per the Peto-Prentice test). We interpret this as an evidence for different evolutionary paths in higher mass discs. Further observations are required to fully determine the low-mass end of the cumulative disc distribution and ascertain whether the populations are truly similar below a mass threshold. Unfortunately, it is possible that the results presented here are biased by completeness in the $0.1-1.0 \mathrm{mJy}$ disc regime, and the low-flux end of the cumulative distributions will be different.

We do not find a qualitative difference if we instead transform the fluxes to match the IC 348 survey using the higher $\alpha=4.4$ value found in Section 3.3. We find $\sigma$ Ori exhibits the largest difference, owing to the relatively small number of discs with $F_{1.1 \mathrm{~mm}}>15 \mathrm{mJy}$. If a larger value of $\alpha$ proves to be generically true between regions, the results in this section still hold.

We are aware that biases exist between the regions that may be corrected for via resampling of the region, but owing to the slight dependence of disc mass and stellar host mass, we opt to instead perform a relative comparison. We do not expect that the resampling would strongly change our results, as the full disc flux distribution comparisons are in line with the findings of previous studies (e.g. Ruíz-Rodríguez et al. 2018).

Taken together, our results suggest that even though inner disc fraction does evolve in the 1-4 Myr time frame, spin-up of stars does not appear to proceed as expected (see Section 4.3) and disc masses around $\log T=3.4-3.6$ stars do not seem to evolve significantly (Section 5.2). In contrast, we see large variance in high-mass discs in that time frame in both the regional comparison and in the previously postulated age-gradient of IC 348 . We therefore have a 


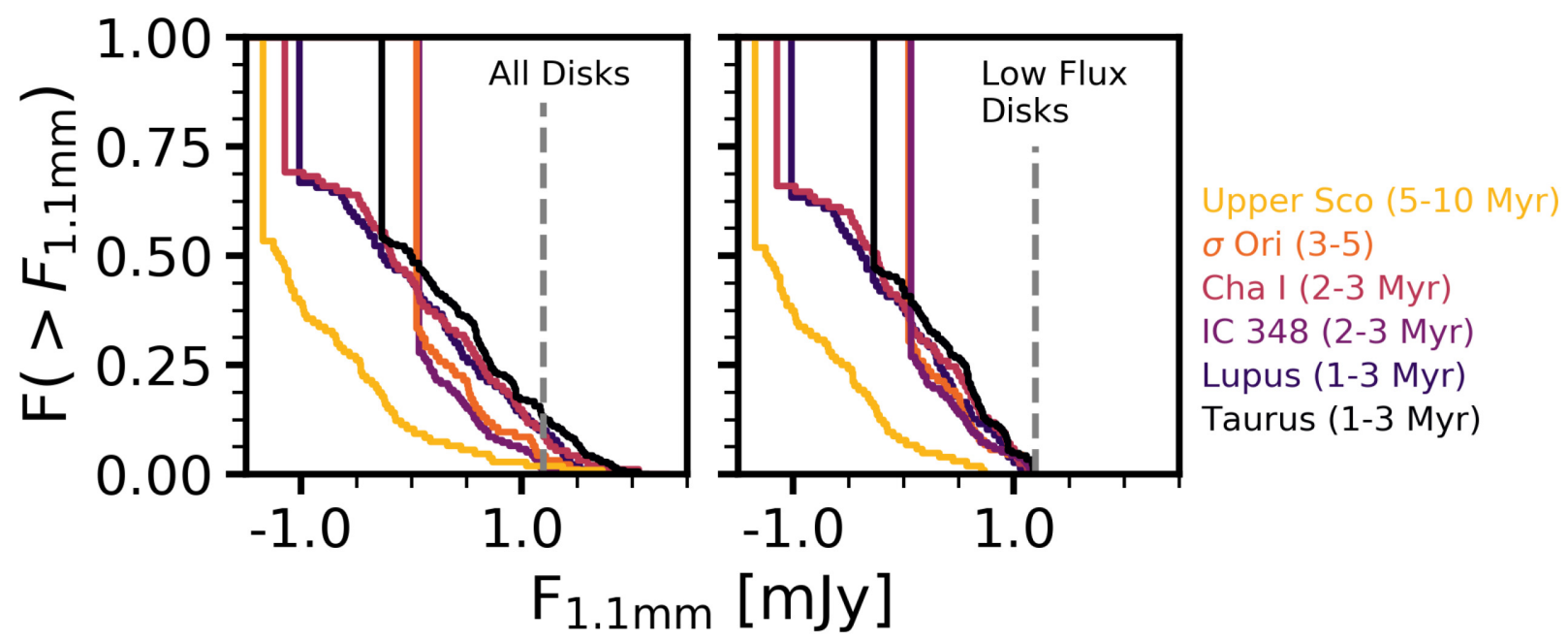

Figure 11. Cumulative distribution functions for different star-forming regions, normalized to the distance and wavelength of the LMT IC 348 sample ( $\lambda=$ $1.1 \mathrm{~mm}, d=320 \mathrm{pc}$ ). The left-hand panel shows the total cumulative distribution function for each region, colour-coded as the key at right. The populations are clearly different, and appear to follow a rough relation with age. The right-hand panel shows the same cumulative distribution functions, except with the most massive discs (>15 mJy) removed. In this case, all star-forming regions except the older Upper Sco are indistinguishable. The vertical dashed line in each panel indicates $15 \mathrm{mJy}$.

Table 6. Table of $p_{\emptyset}$ values for different clusters, with and without higher mass discs.

\begin{tabular}{lcc}
\hline Region & $p_{\emptyset, \text { all }}$ & $p_{\emptyset, F<15 \mathrm{mJy}}$ \\
\hline Taurus & 0.006 & 0.182 \\
Lupus & 0.002 & 0.046 \\
Cha I & 0.001 & 0.018 \\
$\sigma$ Ori & 0.482 & 0.777 \\
Upper Sco & 0.080 & 0.042 \\
\hline
\end{tabular}

fair amount of evidence that higher mass discs evolve faster $(\approx 1 \mathrm{Myr}$ time-scales) than low-mass discs $(\approx 5 \mathrm{Myr})$, in agreement with the typically lower inner disc fraction of higher mass stars relative to lower mass siblings in clusters.

Assuming a fixed surface density profile, high-mass discs are large radius discs, and thus much of their mass excess is weakly bound and prone to much faster photoevaporative mass-loss than a low-mass disc around a similar mass star (e.g. Adams et al. 2006). With viscous disc evolution and similar densities between discs, the assertion that high-mass discs are younger suggests that either (a) high-mass discs are smaller relative to low-mass discs, or (b) the viscosity in high-mass discs is larger than that in low-mass discs, or (c) giant planet formation is likely to be more efficient in the massive discs, which could result in faster evolution of the disc mass, even at relatively young ages for the clusters. Parker \& Alves de Oliveira (2017) found no evidence for mass segregation of stars in IC 348, further finding that a computed initial density of IC 348 suggests that dynamical interactions could have affected some small percentage of binaries and protoplanetary discs. We do not confirm or rule out such interactions, but do affirm that dynamical interactions are unlikely to be the dominant cause of the observed disc masses.

Our findings allow for both the photoevaporative scenario and smaller radii for high-mass discs (in accordance with lower viscous lifetimes) to set the lifetime of a given disc. The anticorrelation of higher mass discs with proximity to the B5 binary in IC 348 suggests that high-mass discs in clusters may suffer mass-loss long before secular evolution has time to occur. Thus, in order to make progress on characterizing the evolution of different mass discs, future work must find ways to constrain the relative impact of regional environmental effects on discs on the characterization of secular disc evolution, including multiwavelength and resolved observations to break modelling degeneracies.

\subsection{Comparison to Taurus discs}

Through various means, most analyses in the literature have shown that IC 348 is composed of a more evolved set of protoplanetary discs than those in Taurus, consistent with their isochronal age difference of a few million years (for example, the relative amount of excess IR emission is lower in IC 348 than Taurus; Lada et al. 2006). In addition, the fraction of known members that possess IR excess emission is lower on average in IC 348 (e.g. Haisch et al. 2001). Based on Rebull et al. (2010), Taurus has younger IR signatures, including a 70 per cent (173/245) disc fraction and Class II to Class I ratio of 2.76 (127/46), as compared to the Gutermuth et al. (2009) values for IC 348 , which has a 37 per cent (160/431) disc fraction (computed from the combined Gutermuth et al. 2009 and IN-SYNC; Cottaar et al. 2014 samples) and a 9.0 (144/16) Class II to Class I ratio. Until recently, however, insufficient numbers of IC 348 discs had been detected in mm-waves to enable a fair comparison of the disc masses of these two regions.

Unlike the empirical calibration in Section 3.3, we elect to use the dust emissivity reported in Andrews et al. (2013), resulting in a flux scaling of $F_{v} \propto v^{\alpha}$, where $\alpha=2.4$. We choose this dust emissivity for the Taurus data owing to the preponderance of data for individual sources in the region. The chosen dust emissivity results in a flux scaling of $F_{1.1 \mathrm{~mm}}=1.49 F_{1.3 \mathrm{~mm}}$. We scale the homogeneous list of $1.3 \mathrm{~mm}$ fluxes in Andrews et al. (2013) using this relation. Additionally, we place Taurus at the distance of IC 348 using the canonical distances for each: $F_{320 \mathrm{pc}}=\left(\frac{140^{2}}{320^{2}}\right) F_{140 \mathrm{pc}}=$ $0.191 F_{140 \mathrm{pc}}$.

For IC 348, we adopt the uniform set of spectroscopically derived stellar parameters from the IN-SYNC survey (Cottaar et al. 2014). For Taurus, we adopt corresponding values derived from archival 


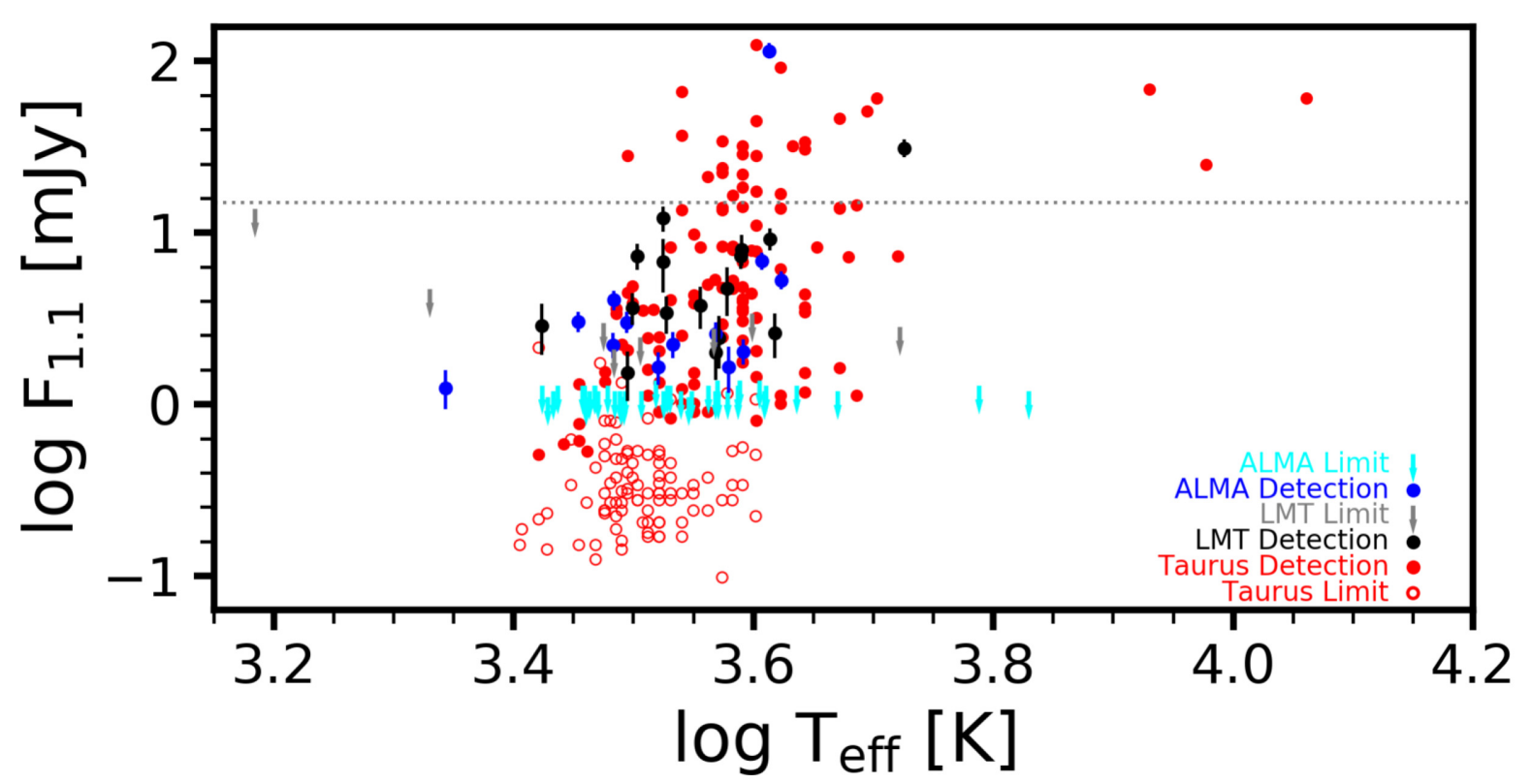

Figure 12. Comparison of IC 348 sources on the flux-effective temperature plane to those of Taurus. The dashed grey line indicates a flux of 15 mJy.

data in Andrews et al. (2013). In Fig. 12, we plot the distributions of disc flux (or lower limit) with stellar temperature. In both Taurus and IC 348, the locus of disc fluxes for stars with $3.4<\log T<$ 3.6 is in reasonable agreement in the range where both regions are well surveyed (i.e. converted fluxes $>3 \mathrm{mJy}$ ). The primary difference between Taurus and IC 348 disc fluxes becomes clear at higher stellar temperatures $(\log T>3.6)$, a distinction that has been observed in the disc fractions of both as well (Lada et al. 2006). The dependence of disc evolution on stellar mass leaves open whether the discs within an apparently less evolved mass range are substantially different or not. The results presented here suggest that there appears to be a great deal of similarity among the distribution of disc fluxes between these two regions, and thus a stability of sorts in the evolution of discs around late-type stars that seems to last through their first few Myr, at least.

\section{SUMMARY}

We present the results of a snapshot $\lambda=1.1 \mathrm{~mm}$ survey with the LMT and AzTEC. We measure the continuum emission from 30 discs (including two new candidate TDs), and place upper limits on 88 others in the IC 348 star-forming region. Coupled with the results of Ruíz-Rodríguez et al. (2018), a total of 44 IR excess sources from Gutermuth et al. (2009) have millimetre detections in IC 348, along with 97 upper limits.

The main scientific results of this paper are as follows:

(1) The IC 348 region hosts a number of moderate-mass dusty discs around Class II objects, with no clear dependence on stellar surface temperatures.

(2) The presence or absence of massive discs in IC 348 depends on location within the region if we partition the sources according to interior/exterior or according to north/south. The difference is more pronounced in the north/south partition than in the interior/exterior partition, with the southern half hosting appreciably more massive discs.
(3) Combining our observations with Ruíz-Rodríguez et al. (2018), we find modest evidence for a significantly higher dust emissivity exponent than previously empirically determined in Taurus, $\beta_{\text {IC } 348}=2.61 \pm 1.24$. The low significance of the data value suggests that further work is needed characterizing the dust emissivity exponent in IC 348 and other regions apart from Taurus.

(4) Two previously unknown candidate transitions discs were serendipitously detected, potentially increasing the number of known TDs from 10 to 12 (following the sample of Gutermuth et al. 2009).

(5) IC 348 stellar hosts show a similar rotation rate signature to that of the Orion star-forming region. The presence of IR excess discs inhibits fast stellar rotation. The presence or absence of significant mm emission does not appear to influence the relation.

(6) For similar stellar hosts, discs in IC 348 are comparable to discs in Taurus. However, by number, Taurus hosts many more high-flux discs than IC 348, and further contains more massive stars, making an overall comparison of the regions complicated.

(7) While the cumulative disc mass function varies strongly between regions, when the disc mass functions are flux- (mass-) limited, the regions largely resemble one another in their disc mass functions. Further observations are required to clarify where and why differences between regions are important.

Future work in the region is needed in order to complete the sample. In the short term, 21 sources from the IR excess list of Gutermuth et al. (2009) have yet to be observed in IC 348. In the longer term, the region would benefit from a deep, blind $\mathrm{mm}$ survey to detect any additional sources of emission such as the serendipitous candidate TDs presented in this paper.

\section{ACKNOWLEDGEMENTS}

We thank the anonymous referee for comments which strengthened the paper. We thank Suzan Edwards, Luisa Rebull, Kevin Covey, James Muzerolle, Fred Adams, Tom Megeath, and Kim WardDuong for a close reading of the manuscript and comments that significantly improved the work. We thank Sean Andrews for 
discussions that shaped the early direction of this work. RAG's participation in this project was supported by NASA ADAP grants NNX11AD14G, NNX15AF05G, and NNX17AF24G, and NSF grant AST 1636621 in support of TolTEC, the next generation mm-wave camera for LMT. The AzTEC instrument was built and operated through support from NSF grant 0504852 to the Five College Radio Astronomy Observatory. The authors gratefully acknowledge the many contributions of David Hughes in leading the LMT to its successful operational state. The authors also thank the personnel and observers at the LMT that helped obtain the observations, including Ricardo Chavez, Victor Gomez, Alfredo Montaña, Erica Keller, Arturo Gomez, James Lowenthal, David Sánchez, Jesús Rivera, Mihwa Han, Miguel Chávez, Fabian Rosales, Jorge Zavala, Dev Raj, David Sánchez, Ivan Rodríguez, Raúl Maldonado, Jesús Contreras, and Salvador Ventura. This work is based in part on observations made with the Spitzer Space Telescope, which is operated by the Jet Propulsion Laboratory, California Institute of Technology, under a contract with NASA. This research used Astropy, a community-developed core PYTHON package for Astronomy (The Astropy Collaboration et al. 2018). This work has used data from the European Space Agency (ESA) mission Gaia (https://www.cosmos.esa.int/gaia), processed by the Gaia Data Processing and Analysis Consortium (DPAC, https: //www.cosmos.esa.int/web/gaia/dpac/consortium). Funding for the DPAC has been provided by national institutions, in particular the institutions participating in the Gaia Multilateral Agreement.

\section{REFERENCES}

Adams F. C., Proszkow E. M., Fatuzzo M., Myers P. C., 2006, ApJ, 641, 504

Alexander R., Pascucci I., Andrews S., Armitage P., Cieza L., 2014, Protostars and Planets VI. University of Arizona Press, Tucson, p. 475

Allard F., Homeier D., Freytag B., 2011, in Johns-Krull C., Browning M. K., West A. A., eds, ASP Conf. Ser., Vol. 448, Model Atmospheres From Very Low Mass Stars to Brown Dwarfs. Astron. Soc. Pac., San Francisco, p. 91

Andrews S. M., Williams J. P., 2005, ApJ, 631, 1134

Andrews S. M., Williams J. P., 2007, ApJ, 659, 705

Andrews S. M., Rosenfeld K. A., Kraus A. L., Wilner D. J., 2013, ApJ, 771, 129

Ansdell M. et al., 2016, ApJ, 828, 46

Ansdell M., Williams J. P., Manara C. F., Miotello A., Facchini S., van der Marel N., Testi L., van Dishoeck E. F., 2017, AJ, 153, 240

Astropy Collaboration, 2018, AJ, 156, 123

Baraffe I., Homeier D., Allard F., Chabrier G., 2015, A\&A, 577, A42

Barenfeld S. A., Carpenter J. M., Ricci L., Isella A., 2016, ApJ, 827, 142

Barenfeld S. A., Carpenter J. M., Sargent A. I., Isella A., Ricci L., 2017, ApJ, 851, 85

Bary J. S., Petersen M. S., 2014, ApJ, 792, 64

Beckwith S. V. W., Sargent A. I., Chini R. S., Guesten R., 1990, AJ, 99, 924

Bell C. P. M., Naylor T., Mayne N. J., Jeffries R. D., Littlefair S. P., 2013, MNRAS, 434, 806

Bryan S. et al., 2018, in Zmuidzinas J., Gao J. -R., eds, Proc. SPIE Conf. Ser.Vol. 10708, Millimeter, Submillimeter, and Far-Infrared Detectors and Instrumentation for Astronomy IX. SPIE, Bellingham, p. 107080J

Cieza L., Baliber N., 2006, ApJ, 649, 862

Cieza L., Baliber N., 2007, ApJ, 671, 605

Cieza L., Williams J., Kourkchi E., Andrews S., Casassus S., Graves S., Schreiber M. R., 2015, MNRAS, 454, 1909

Cottaar M. et al., 2014, ApJ, 794, 125

Cottaar M. et al., 2015, ApJ, 807, 27

Da Rio N. et al., 2016, ApJ, 818, 59
Eisenstein D. J. et al., 2011, AJ, 142, 72

Eisner J. A. et al., 2018, ApJ, 860, 77

Eisner J. A., Bally J. M., Ginsburg A., Sheehan P. D., 2016, ApJ, 826, 16

Espaillat C. et al., 2012, ApJ, 747, 103

Espaillat C. et al., 2014, Protostars and Planets VI. University of Arizona Press, Tucson, p. 497

Espaillat C., Furlan E., D’Alessio P., Sargent B., Nagel E., Calvet N., Watson D. M., Muzerolle J., 2011, ApJ, 728, 49

Evans N. J., II et al., 2009, ApJS, 181, 321

Feigelson E. D., Nelson P. I., 1985, ApJ, 293, 192

Fernandez M. A. et al., 2017, PASP, 129, 084201

Gaia Collaboration, 2016, A\&A, 595, A1

Gaia Collaboration, 2018, A\&A, 616, A1

Guarcello M. G. et al., 2016, preprint (arXiv:1605.01773)

Gutermuth R. A., Heyer M., 2015, AJ, 149, 64

Gutermuth R. A., Megeath S. T., Myers P. C., Allen L. E., Pipher J. L., Fazio G. G., 2009, ApJS, 184, 18

Haisch Jr. K. E., Lada E. A., Lada C. J., 2001, ApJ, 553, L153

Herbst W., Maley J. A., Williams E. C., 2000, AJ, 120, 349

Herbst W., Bailer-Jones C. A. L., Mundt R., 2001, ApJ, 554, L197

Hildebrand R. H., 1983, Q. J. R. Astron. Soc., 24, 267

Hillenbrand L. A., 2005, in Livio, M., Sahu, K., Valenti, J., eds, A Decade of Extrasolar Planets around Normal Stars. Cambridge University Press, Cambridge, p. 8

Jørgensen J. K. et al., 2006, ApJ, 645, 1246

Jørgensen J. K., Johnstone D., Kirk H., Myers P. C., Allen L. E., Shirley Y. L., 2008, ApJ, 683, 822

Lada C. J. et al., 2006, AJ, 131, 1574

Lee N., Williams J. P., Cieza L. A., 2011, ApJ, 736, 135

Luhman K. L., Stauffer J. R., Muench A. A., Rieke G. H., Lada E. A., Bouvier J., Lada C. J., 2003, ApJ, 593, 1093

Mamajek E. E., 2009, in Usuda T., Tamura M., Ishii M., eds, AIP Conf. Proc.Vol. 1158, Initial Conditions of Planet Formation: Lifetimes of Primordial Disks. Am. Inst. Phys., New York, p. 3

Megeath S. T. et al., 2012, AJ, 144, 192

Muench A. A., Lada C. J., Luhman K. L., Muzerolle J., Young E., 2007, AJ, 134,411

Muzerolle J., Allen L. E., Megeath S. T., Hernández J., Gutermuth R. A., 2010, ApJ, 708, 1107

Ortiz-León G. N. et al., 2018, ApJ, 865, 73

Parker R. J., Alves de Oliveira C., 2017, MNRAS, 468, 4340

Pascucci I. et al., 2016, ApJ, 831, 125

Pfalzner S., Steinhausen M., Menten K., 2014, ApJ, 793, L34

Purcell E. M., 1969, ApJ, 158, 433

Rayner J. T., Cushing M. C., Vacca W. D., 2009, ApJS, 185, 289

Rebull L. M., Stauffer J. R., Megeath S. T., Hora J. L., Hartmann L., 2006 ApJ, 646, 297

Rebull L. M. et al., 2007, ApJS, 171, 447

Rebull L. M. et al., 2010, ApJS, 186, 259

Rieke G. H., Lebofsky M. J., 1985, ApJ, 288, 618

Ripepi V. et al., 2014, MNRAS, 437, 906

Ruíz-Rodríguez D. et al., 2018, MNRAS, 478, 3674

Scott K. S. et al., 2008, MNRAS, 385, 2225

Skrutskie M. F. et al., 2006, AJ, 131, 1163

Sokol A.D. et al., 2019, MNRAS, 483, 407

Strom K. M., Strom S. E., Edwards S., Cabrit S., Skrutskie M. F., 1989, AJ, 97,1451

Ward-Duong K. et al., 2018, AJ, 155, 54

Williams J. P., Cieza L. A., 2011, ARA\&A, 49, 67

Wilson G. W. et al., 2008, MNRAS, 386, 807

Yao Y., Meyer M. R., Covey K. R., Tan J. C., Da Rio N., 2018, ApJ, 869, 72

Zasowski G. et al., 2013, AJ, 146, 81

This paper has been typeset from a $\mathrm{T}_{\mathrm{E}} \mathrm{X} / \mathrm{L} \mathrm{AT} \mathrm{E} \mathrm{X}$ file prepared by the author. 